EUROPEAN CENTRAL BANK

WORKING PAPER SERIES

NO. 32 I / MARCH 2004

FREQUENCY DOMAIN PRINCIPAL COMPONENTS ESTIMATION OF FRACTIONALLY COINTEGRATED PROCESSES

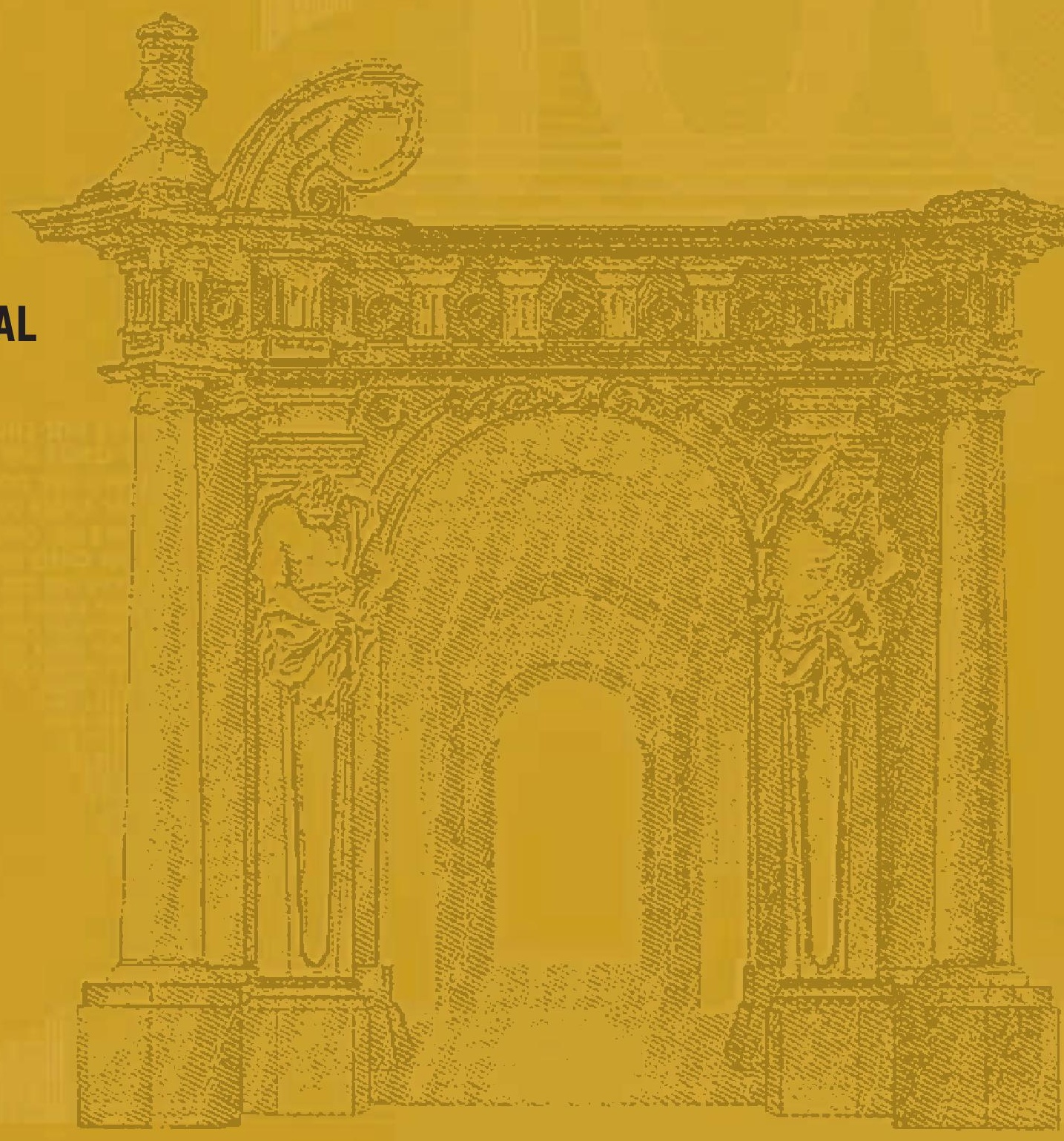




\section{FREQUENCY DOMAIN PRINCIPAL COMPONENTS ESTIMATION OF FRACTIONALLY COINTEGRATED PROCESSES'}

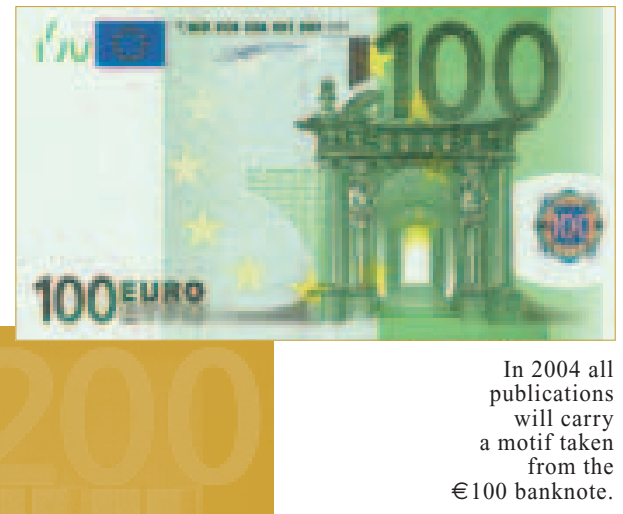

by Claudio Morana ${ }^{2}$

This paper can be downloaded without charge from http://www.ecb.int or from the Social Science Research Network electronic library at http://ssrn.com/abstract_id $=526984$. 


\section{드 European Central Bank, 2004}

\section{Address}

Kaiserstrasse 29

60311 Frankfurt am Main, Germany

Postal address

Postfach 160319

60066 Frankfurt am Main, Germany

Telephone

+496913440

\section{Internet}

http://www.ecb.int

Fax

+496913446000

Telex

411144 ecb d

All rights reserved.

Reproduction for educational and noncommercial purposes is permitted provided that the source is acknowledged.

The views expressed in this paper do not necessarily reflect those of the European Central Bank.

The statement of purpose for the ECB Working Paper Series is available from the ECB website, http://www.ecb.int.

ISSN 1561-0810 (print)

ISSN 1725-2806 (online) 


\section{CONTENTS}

Abstract 4

Non-technical summary 5

1 Introduction 6

2 Definitions of long memory 7

3 Spectral properties for vector fractionally cointegrated processes $\quad 8$

3.1 The frequency domain principal components estimator

4 Additional spectral properties for vector fractionally cointegrated processes

5 Monte Carlo results 24

6 Empirical applications 26

6.1 Long memory processes 26

$6.2 \mathrm{I}(1)$ processes $\quad 28$

7 Conclusions 28

8 Appendix 29

References 34

Tables and figures $\quad 37$

European Central Bank

working paper series 


\begin{abstract}
In this paper we study the zero frequency spectral properties of fractionally cointegrated long memory processes and introduce a new frequency domain principal components estimator of the cointegration space and the factor loading matrix for the long memory factors. We find that for fractionally differenced (fractionally) cointegrated processes the squared multiple coherence at the zero frequency is equal to one, the spectral density matrix at the zero frequency is singular, and the factor loading and cointegrating matrices can be obtained from the eigenvectors of the spectral matrix at the zero frequency, associated with the positive and zero roots, respectively. A Monte Carlo simulation reveals that the proposed principal components estimator has already good properties with relatively small sample sizes.

Keywords: Fractional cointegration, long memory, frequency domain analysis.

J.E.L: C22
\end{abstract}




\section{Non-technical summary}

As shown by Phillips (1986) and Robinson and Yajima (2002), for the case of I(1) and long memory processes $(\mathrm{I}(\mathrm{d}) 0<\mathrm{d}<0.5)$, respectively, cointegration implies that the spectral matrix of the differenced processes at the zero frequency is of reduced rank, equal to the number of common persistent (I(1), I(d)) factors in the system. Phillips (1986) and Phillips and Ouliaris (1988) have also shown that the number of zero eigenvalues of the spectral matrix of the differenced series at the zero frequency is equal to the number of cointegrating relationships, and that the cointegrating vectors are the associated eigenvectors. In the paper we show that an estimator of the factor loading and cointegrating matrices can be obtained from the eigenvectors of the spectral matrix of the fractionally differenced processes at the zero frequency, associated with the positive and zero roots, respectively. While the results in this paper are derived for long memory processes, the same conclusions apply for the case of cointegrated I(1) processes. This follows from the definition of cointegration adopted, which is the same as the one employed by Engle and Granger (1987). In the paper we exploit this important result and propose a frequency domain principal components estimator of the cointegration space and the factor loading matrix for the common long memory factors, which can then be estimated using the Kasa (1992) decomposition. We also show that an equivalent approach can be implemented using the series in levels as the frequency tends to zero. A Monte Carlo simulation shows that the proposed estimator has good properties already with relatively small sample sizes. In the paper we also show that the multiple squared coherence at the zero frequency for fractionally differenced (fractionally) cointegrated processes is equal to one, while the simple squared coherences assume a value greater than zero but lower than one. In the bivariate case the multiple and simple squared coherence coincide and, therefore, the simple squared coherence at the zero frequency assumes a unitary value. These results extend the previous findings of Granger and Weiss (1983) and Levy (2002) for I(1) processes. We also find that processes that are not fractionally cointegrated show in general positive, but lower than one, multiple and simple squared coherences at the zero frequency. In the case the dependent and independent variables are driven by different long memory factors, i.e. in the case the dependent variable is orthogonal at the zero frequency to any of the regressors, the squared multiple coherence will assume a zero value, as any of the squared simple coherences. We finally show that all the above results also hold for the series in levels, as the frequency tends to zero. In addition to be of theoretical interest, all the properties derived in the paper may be exploited in practice, as is shown in the two empirical applications which conclude the paper. 


\section{Introduction}

As shown by Phillips (1986) and Robinson and Yajima (2002), for the case of $\mathrm{I}(1)$ and long memory processes $(\mathrm{I}(\mathrm{d}) 0<d<0.5)$, respectively, cointegration implies that the spectral matrix of the differenced processes at the zero frequency is of reduced rank, equal to the number of common persistent (I(1), I(d)) factors in the system. ${ }^{1}$ Phillips (1986) and Phillips and Ouliaris (1988) have also shown that the number of zero eigenvalues of the spectral matrix of the differenced series at the zero frequency is equal to the number of cointegrating relationships, and that the cointegrating vectors are the associated eigenvectors. In the paper we show that an estimator of the factor loading and cointegrating matrices can be obtained from the eigenvectors of the spectral matrix of the fractionally differenced processes at the zero frequency, associated with the positive and zero roots, respectively. While the results in this paper are derived for long memory processes, the same conclusions apply for the case of cointegrated I(1) processes. This follows from the definition of cointegration adopted, which is the same as the one employed by Engle and Granger (1987). In the paper we exploit this important result and propose a frequency domain principal components estimator of the cointegration space and the factor loading matrix for the common long memory factors, which can then be estimated using the Kasa (1992) decomposition. We also show that an equivalent approach can be implemented using the series in levels as the frequency tends to zero. A Monte Carlo simulation shows that the proposed estimator has good properties already with relatively small sample sizes. In the paper we also show that the multiple squared coherence at the zero frequency for fractionally differenced (fractionally) cointegrated processes is equal to one, while the simple squared coherences assume a value greater than zero but lower than one. In the bivariate case the multiple and simple squared coherence coincide and, therefore, the simple squared coherence at the zero frequency assumes a unitary value. These results extend the previous findings of Granger and Weiss (1983) and Levy (2002) for I(1) processes. ${ }^{2}$ We also find that processes that are not fractionally cointegrated show in general positive, but lower than one, multiple and simple squared coherences at the zero frequency. In the case the dependent and independent

\footnotetext{
${ }^{1}$ Robinson and Yajima (2002) have demonstrated this result as the frequency tends to zero. Marinucci and Robinson (1998) have also shown that this result holds for the series in levels. The two results are related since for the $\mathrm{I}(\mathrm{d})$ vector process $\mathbf{x}_{t} \mathbf{f}_{\mathbf{x}}(\omega) \sim \omega^{-2 d} \mathbf{G}$ $\omega \rightarrow 0^{+}$and $\mathbf{f}_{\Delta^{d} \mathbf{x}}(\omega) \sim \mathbf{G} \omega \rightarrow 0^{+}$.

${ }^{2}$ As noted by Granger and Weiss (1983), these results are valid in general for the CI(d,0) case. In the paper we also show that the same results hold for the $\mathrm{CI}(\mathrm{d}, \mathrm{b})$ case, $b>0$ $d-b>0$.
} 
variables are driven by different long memory factors, i.e. in the case the dependent variable is orthogonal at the zero frequency to any of the regressors, the squared multiple coherence will assume a zero value, as any of the squared simple coherences. We finally show that all the above results also hold for the series in levels, as the frequency tends to zero. In addition to be of theoretical interest, these properties may be exploited in practice in empirical analysis.

The plan of the paper is as follows. In section two we provide definitions of long memory processes. In sections three and four we introduce the frequency domain principal components estimator and investigate the spectral properties of fractionally cointegrated processes. In section five we present the results of a Monte Carlo exercise aiming to evaluate the small sample properties of the proposed estimator. Finally in section six we provide applications with real data and in section seven we conclude.

\section{Definitions of long memory}

There are two main definitions of long memory. Firstly, a stationary processes $x_{t}$ is said to be long range dependent if the autocorrelation function $\left(\gamma_{x}(\tau)\right)$ is significantly different from zero at very long lags, that is

$$
\gamma_{x}(\tau)=c_{\rho} \tau^{2 d-1}
$$

where $c_{\rho}$ is a positive constant, $\tau$ is the order of the autocorrelation and $d \in(0,0.5)$ is the coefficient of fractional integration. Differently from $\mathrm{I}(0)$ processes, $\mathrm{I}(\mathrm{d})$ stationary processes do not show an exponentially fast decay of the autocorrelation function, but a slow hyperbolic decay. An equivalent definition in the frequency domain is

$$
f_{x}(\omega)=c_{f} \omega^{-2 d} \text { as } \omega \rightarrow 0^{+}
$$

where $c_{f}$ is a positive constant, $f_{x}(\omega)$ is the spectrum and $\omega$ is the frequency in radians. Therefore, in the frequency domain, long memory is detected on the basis of the presence of a singular point in the spectrum at the zero frequency.

Hence, similarly to I(1) processes, long memory processes have non definite spectral density at the zero frequency, but similarly to $\mathrm{I}(0)$ processes the effects of shocks eventually die out, although at a very slow hyperbolic 
rate. It is the strong shock persistence of long memory processes that justify cointegration analysis for stationary processes.

In the paper we assume that all the long memory processes share the same order of integration $d$, and we consider the $\mathrm{I}(d)-\mathrm{I}(0)$ case. Hence, the definition of fractional cointegration follows Engle and Granger (1987), namely $p$ long memory processes are fractionally cointegrated if there exists at least one linear combination which is $\mathrm{I}(0)$, i.e. $x_{1, t}, x_{2, t}, \ldots, x_{p, t} \sim C I(d, 0) d \in(0,0.5)$. While for long memory processes other definitions of cointegration can be envisaged (Robinson and Yajima, 2002), the case considered in this paper is likely to be of empirical relevance, and is an important benchmark in any case. However, we show that our results extend also to the general case $C I(d, b) b>0 d-b>0$, i.e. where the cointegrating residuals still show long memory, although their degree of integration is lower than the one of the actual series.

\section{Spectral properties for vector fractionally cointegrated processes}

Let us assume the following common long memory factor model

$$
\begin{aligned}
\mathbf{x}_{t} & =\Theta \boldsymbol{\mu}_{t}+\mathbf{u}_{t} \\
\Delta^{d} \boldsymbol{\mu}_{t} & =\varepsilon_{t},
\end{aligned}
$$

where $\mathbf{x}_{t}$ is a $p \times 1$ vector of observations on the $p$ fractionally cointegrated long memory processes, $\Theta$ is the $p \times k$ factor loading matrix with $k<p, \boldsymbol{\mu}_{t}$ is a $k \times 1$ vector of observations on the long memory factors (I(d) $0<d<0.5$ ), $\varepsilon_{t} \sim$ i.i.d. $\left(\mathbf{0}, \Sigma_{\varepsilon}\right)$ with dimension $k \times 1$ and $\Sigma_{\varepsilon}=\mathbf{I}_{k}, \mathbf{u}_{t}$ is a $p \times 1$ vector of observations on the $\mathrm{I}(0)$ components with $\Phi(L) \mathbf{u}_{t}=\Omega(L) \mathbf{v}_{t}$, all the roots of the polynomial matrices in the lag operator $\Phi(L)$ and $\Omega(L)$ are outside the unit circle, and $\mathbf{v}_{t} \sim$ i.i.d. $\left(\mathbf{0}, \Sigma_{u}\right)$ with dimension $p \times 1$.

Applying fractional differencing to [1], yields

$$
\Delta^{d} \mathbf{x}_{t}=\Theta \varepsilon_{t}+\Delta^{d} \mathbf{u}_{t}
$$

Then the autocovariance and cross covariance functions can be computed as $\Gamma(\tau)=E\left[\left(\Delta^{d} \mathbf{x}_{t+\tau}-E\left[\Delta^{d} \mathbf{x}_{t}\right] \quad \Delta^{d} \mathbf{x}_{t}-E\left[\Delta^{d} \mathbf{x}_{t}\right]\right)^{\prime}\right]$, yielding 


$$
\begin{aligned}
\Gamma(\tau) & =E\left[\left(\Theta \varepsilon_{t+\tau}+\Delta^{d} \mathbf{u}_{t+\tau}\right)\left(\Theta \varepsilon_{t}+\Delta^{d} \mathbf{u}_{t}\right)^{\prime}\right] \\
& =E\left[\Theta \varepsilon_{t+\tau} \varepsilon_{t}^{\prime} \Theta^{\prime}\right]+E\left[\Theta \varepsilon_{t+\tau} \Delta^{d} \mathbf{u}_{t}^{\prime}\right]+E\left[\Delta^{d} \mathbf{u}_{t+\tau} \varepsilon_{t}^{\prime} \Theta^{\prime}\right]+E\left[\Delta^{d} \mathbf{u}_{t+\tau} \Delta^{d} \mathbf{u}_{t}^{\prime}\right] \\
& =\Theta \Gamma_{\varepsilon}(\tau) \Theta^{\prime}+\Theta \Gamma_{\varepsilon, \Delta^{d} u^{\prime}}(\tau)+\Gamma_{\Delta^{d} u, \varepsilon^{\prime}}(\tau) \Theta^{\prime}+\Gamma_{\Delta^{d} u}(\tau)
\end{aligned}
$$

Applying the definitions for the spectrum and cross spectrum to the elements in [3], the spectral density matrix for the fractionally differenced processes

$$
\mathbf{f}(\omega)=\left[\begin{array}{cccc}
f_{\Delta^{d} x_{1}}(\omega) & f_{\Delta^{d} x_{1} \Delta^{d} x_{2}}(\omega) & \ldots & f_{\Delta^{d} x_{1} \Delta^{d} x_{p}}(\omega) \\
f_{\Delta^{d} x_{2} \Delta^{d} x_{1}}(\omega) & f_{\Delta^{d} x_{2}}(\omega) & \vdots & \vdots \\
\vdots & \vdots & \ddots & \vdots \\
f_{\Delta^{d} x_{p} \Delta^{d} x_{1}}(\omega) & \cdots & \cdots & f_{\Delta^{d} x_{p}}(\omega)
\end{array}\right]
$$

where $\omega$ measures the frequency in radians, $f_{\Delta^{d} x_{i}}(\omega)=\frac{1}{2 \pi} \int_{-\infty}^{+\infty} \gamma_{\Delta^{d} x_{i}}(\tau) e^{-i \omega} d \tau$ is the spectral density function for the $i$ th fractionally differenced process, $f_{\Delta^{d} x_{i} \Delta^{d} x_{j}}(\omega)=\frac{1}{2 \pi} \int_{-\infty}^{+\infty} \gamma_{\Delta^{d} x_{i} \Delta^{d} x_{j}}(\tau) e^{-i \omega} d \tau$ is the cross spectral density function for the fractionally differenced processes $i$ and $j, \gamma_{\Delta^{d} x_{i}}(\tau)$ denote the autocovariance function, and $\gamma_{\Delta^{d} x \Delta^{d} y}(\tau)$ is the cross covariance function, can be written as

$$
\mathbf{f}(\omega)=\Theta \mathbf{f}_{\varepsilon}(\omega) \Theta^{\prime}+\Theta \mathbf{f}_{\varepsilon, \Delta^{d} u^{\prime}}(\omega)+\mathbf{f}_{\Delta^{d} u, \varepsilon^{\prime}}(\omega) \Theta^{\prime}+\mathbf{f}_{\Delta^{d} u}(\omega),
$$

where the $\mathbf{f}_{i}(\omega)$ matrices contain the spectral and cross spectral functions for the given vectors, evaluated at the frequency $\omega$. By noting that

$$
\begin{aligned}
& \text { 1) } \mathbf{f}_{\varepsilon}(\omega)=\frac{1}{2 \pi} \Sigma_{\varepsilon}-\pi \leq \omega \leq \pi \text { since } \varepsilon_{j} \sim i . i . d j=1, \ldots, k ; \\
& \text { 2) } f_{\Delta^{d} u_{i}}(\omega)=\left|1-e^{-i \omega}\right|^{2 d} f_{u_{i}}(\omega)=[2 \sin (\omega / 2)]^{2 d} f_{u_{i}}(\omega) i=1, \ldots, p ; \\
& f_{\Delta^{d} u_{i}, \Delta^{d} u_{j}}(\omega)=\left|1-e^{-i \omega}\right|^{2 d} f_{u_{i}, u_{j}}(\omega)=W\left(e^{-i \omega}\right)\left|1-e^{-i \omega}\right|^{2 d} f_{u_{j}}(\omega)= \\
& \sum_{h=-q}^{s} w_{h} \cos (\omega h)[2 \sin (\omega / 2)]^{2 d} f_{u_{j}}(\omega) i, j=1, \ldots, p i \neq j .
\end{aligned}
$$

Hence $\mathbf{f}_{\Delta^{d} u}(0)=\mathbf{0}$, since $u_{n} \sim I(0) n=1, \ldots, p$ and has a finite spectrum at frequency zero, $\cos (0)=1, \sin (0)=0$, i.e. since $\Delta^{d} u_{n} \sim I(-d) n=1, \ldots, p$, i.e. $\Delta^{d} u_{n}$ is an antipersistent process; 
3) $\mathbf{f}_{\varepsilon, \Delta^{d} u^{\prime}}(0)=\mathbf{0}, \mathbf{f}_{\Delta^{d} u, \varepsilon^{\prime}}(0)=\mathbf{0}$, since $\varepsilon_{j} \sim i . i . d . j=1, \ldots, k, \Delta^{d} u_{n} \sim$ $I(-d) n=1, \ldots, p$, and exploiting the orthonormality property of the matrix $\Sigma_{\varepsilon}\left(\Sigma_{\varepsilon}=\mathbf{I}_{k}\right)$, yields

$$
\mathbf{f}(0)=\frac{1}{2 \pi} \Theta \Theta^{\prime}
$$

Hence $\mathbf{f}(0)$ inherits the properties of the matrix $\Theta^{\prime}$, namely $\mathbf{f}(0)$ is symmetric, it is of reduced rank equal to $k<p$, and it is positive semidefinite. ${ }^{3}{ }^{4}$

\subsection{The frequency domain principal components esti- mator}

Estimation of the factor loading matrix From the symmetry property, it follows that the spectral matrix can be factorised as

$$
2 \pi \mathbf{f}(0)=\mathbf{Q} \Lambda \mathbf{Q}^{\prime}
$$

where $\Lambda$ is the $p \times p$ diagonal matrix of (real) eigenvalues and the matrix $\mathbf{Q}$ is the $p \times p$ matrix of its associated orthogonal eigenvectors. Since $\mathbf{f}(0)$ is of reduced rank $k$, only $k$ eigenvalues are greater than zero. Hence, $\mathbf{Q} \Lambda^{\frac{1}{2}}$ contains $k$ non zero columns and $\Lambda^{\frac{1}{2}} \mathbf{Q}^{\prime} k$ non zero rows. Without lack of generality, by assuming that the eigenvalues are ordered in descending order, the matrix $\mathbf{Q} \Lambda^{\frac{1}{2}}$ can be partitioned as $\left[\left(\begin{array}{c}\mathbf{Q} \Lambda^{\frac{1}{2}} \\ p \times k\end{array}\right)^{*} \underset{p \times(p-k)}{\mathbf{0}}\right]$, so that by the

\footnotetext{
${ }^{3}$ Note that the same results hold for the case in which the $\mathbf{u}$ vector is $\mathrm{I}(\mathrm{b}) b>0 d-b>0$, since $\Delta^{d} \mathbf{u} \sim \mathrm{I}(\mathrm{b}-\mathrm{d})$.

${ }^{4}$ The reduced rank of the spectral matrix for the differenced series was firstly noted by Phillips (1986) and Phillips and Ouliaris (1988) for the I(1) case. Phillips and Ouliaris (1988) have proposed a cointegrating rank test based on the number of non zero eigenvalues of the spectral density matrix at the zero frequency, which provides the number of common trends $k$, and therefore the number of cointegration relationships $r=p-k$. Robinson and Marinucci (1998) and Robinson and Yajima (2002) have shown that a similar result holds for the $\mathrm{I}(\mathrm{d})$ case $(0<d<0.5)$ for the series in levels as the frequency tends to zero, i.e. given the $\mathrm{I}(\mathrm{d})$ vector process $\mathbf{x}_{t}, \mathbf{f}_{\mathbf{x}}(\omega) \sim \omega^{-2 d} \mathbf{G} \omega \rightarrow 0^{+}$, where $\mathbf{G}=\frac{\Theta \Theta^{\prime}}{2 \pi}$. Differently from what is done in these latter papers, by fractionally differencing we work with $\mathrm{I}(0)$ series, and therefore with well defined spectral density functions at the zero frequency. This allows us to establish results exactly at the zero frequency. A fractional cointegrating rank test, based on the number of non zero eigenvalues of the matrix $\mathbf{G}$, has been suggested by Robinson and Yajima (2002), along the lines of Phillips and Ouliaris (1988).
} 
rule of the product of partitioned matrices we have

$$
\left(\underset{p \times p}{\mathbf{Q} \Lambda^{\frac{1}{2}}}\right)\left(\begin{array}{c}
\Lambda_{p \times p}^{\frac{1}{2}} \mathbf{Q}^{\prime} \\
p \times p
\end{array}\right)=\left(\underset{p \times k}{\mathbf{Q} \Lambda^{\frac{1}{2}}}\right)^{*}\left(\begin{array}{c}
\Lambda_{k \times p}^{\frac{1}{2}} \mathbf{Q}^{\prime} \\
{ }^{\prime}
\end{array}\right)^{*}+\underset{p \times p}{\mathbf{0}}=\underset{p \times p}{\Theta \Theta^{\prime}}
$$

The matrix $\left(\mathbf{Q} \Lambda^{\frac{1}{2}}\right)^{*}$ is therefore our estimator of the factor loading ma$\operatorname{trix} \Theta .^{5}$

Interestingly, the procedure implemented has an interpretation in terms of principal components analysis. By construction, the normalised linear combinations $\mathbf{Q}_{i}^{\prime} \Delta^{d} \mathbf{x}^{\prime}$ or $\left(\Lambda^{\frac{1}{2}} \mathbf{Q}^{\prime}\right)_{i}^{*} \Delta^{d} \mathbf{x}^{\prime}, i=1, \ldots, k$, where $\Delta^{d} \mathbf{X}$ is the $T \times p$ matrix of the observations on the $p$ fractionally differenced processes, are the orthogonal linear combinations of the fractionally differenced processes which are characterised by the largest (long-run) variances. ${ }^{6}$

Estimation of the cointegration space Moreover, while the (scaled) eigenvectors, associated with the largest $k$ roots, as shown above, yield an estimate of the factor loadings, the eigenvectors associated with the $p-k$ zero roots yield an estimate of the cointegration space. This result follows from the following consideration. Given the orthogonality property of the eigenvectors, it follows that

$$
\mathbf{Q}_{1, . ., k}^{\prime} \mathbf{Q}_{k+1, . ., p}=\underset{k \times(p-k)}{\mathbf{0}}
$$

where $\mathbf{Q}_{1, . ., k}^{\prime}$ and $\mathbf{Q}_{k+1, . ., p}$ denote the submatrices composed of the $k$ eigenvectors associated with the first $k$ largest roots, and the last $r=p-k$ eigenvectors associated with the zero roots, respectively. Hence $\mathbf{Q}_{k+1, \ldots, p}$ is a right null space basis of the factor loading matrix, which is the definition of the cointegration space, since the cointegration relationships are the linear combinations of the variables which remove the persistent $(\mathrm{I}(\mathrm{d}))$ or permanent $(\mathrm{I}(1))$ component from them (see Engle and Granger, 1987). We can write therefore $\boldsymbol{\beta}=\mathbf{Q}_{k+1, . ., p}$, where $\boldsymbol{\beta}$ denote the $p \times r$ cointegration matrix, obtaining

\footnotetext{
${ }^{5}$ Note that this decomposition is always possible since $\mathbf{f}(0)$ is positive semidefinte, so that the non null eigenvalues are real and positive.

${ }^{6}$ Since for an $\mathrm{I}(\mathrm{d})$ vector process $\mathbf{x}_{t} \mathbf{f}_{\mathbf{x}}(\omega) \sim \omega^{-2 d} \mathbf{G} \omega \rightarrow 0^{+}$the eigenvectors of $\mathbf{f}_{\mathbf{x}}(\omega)$ $\omega \rightarrow 0^{+}$are the same as the eigenvectors of $\mathbf{G}, \mathbf{f}(0)$, or $\Theta \Theta^{\prime}$. Therefore, $\left(\Lambda^{\frac{1}{2}} \mathbf{Q}^{\prime}\right)_{i}^{*} \mathbf{x}^{\prime}$ are the orthogonal linear combinations of the series in levels characterised by the largest (longrun) variances. These linear combinations bear the interpretation of "long-run" principal components. See the section on the interpretation of the proposed estimator.
} 


$$
\boldsymbol{\beta}^{\prime}\left(\mathrm{Q} \Lambda^{\frac{1}{2}}\right)^{*}=\boldsymbol{\beta}^{\prime} \Theta=\underset{r \times k}{\mathbf{0}} \cdot 7
$$

Identification of the factor loading matrix The identification of the factor loading matrix $\Theta$ can be obtained following the approach proposed by King et al. (1991) and Warne (1993) for the identification of the common trends model in time domain. The identification strategy can be implemented as follows.

Let us write the factor loading matrix as

$$
\Theta=\mathbf{Q}_{0}^{*} \rho
$$

where $\boldsymbol{\rho}$ is a $k \times k$ matrix collecting the free parameters in $\Theta$ and $\mathbf{Q}_{0}^{*}$ is the matrix $\left(\mathrm{Q} \Lambda^{\frac{1}{2}}\right)^{*}$ with the identification conditions imposed in such a way that the upper square submatrix of order $k$ is the identity matrix (see Anderson, 1984; p. 556). ${ }^{8}$ This will yield $k(k-1) / 2$ zero restrictions in $\Theta$. From the relationship $\Theta \Theta^{\prime}=2 \pi \mathbf{f}(0)$ we then have

$$
\boldsymbol{\rho} \boldsymbol{\rho}^{\prime}=\left(\mathbf{Q}_{0}^{\prime *} \mathbf{Q}_{0}^{*}\right)^{-1} \mathbf{Q}_{0}^{*}(2 \pi \mathbf{f}(0)) \mathbf{Q}_{0}^{*}\left(\mathbf{Q}_{0}^{\prime *} \mathbf{Q}_{0}^{*}\right)^{-1} .
$$

The matrix $\boldsymbol{\rho} \boldsymbol{\rho}^{\prime}$ is positive definite and symmetric, containing $k(k+1) / 2$ distinct parameters which can be estimated through its Choleski decomposition, leading to a lower triangular $\boldsymbol{\rho}$ matrix and to $k(k+1) / 2$ independent equations. ${ }^{9}$ A total of $k^{2}$ over $p k$ parameters in $\Theta$ will result to be identified using the above discussed procedure. Finally, the remaining $(p-k) k$ parameters will result to be identified by using the condition $\mathbf{Q}_{k+1, \ldots, p}^{\prime} \mathbf{Q}_{0}^{*}=\mathbf{0}$. After estimation the matrix $\Theta$ may be rotated by means of a Givens rotation matrix (Anderson, 1984; p.606) to add further interpretability to the results.

\footnotetext{
${ }^{8}$ Note that the imposition of the identification conditions is always possible. In the case the upper submatrix of dimension $k$ is singular, the identity matrix can be positioned differently in the factor loading matrix, without any consequences for the identification of the model.

${ }^{9}$ Note that a lower triangular $\boldsymbol{\rho}$ matrix does not imply any recursive structure for the factor loading matrix, since the way the common trends affect the vector $\mathbf{x}_{t}$ is determined by the identification condition imposed on $\mathbf{Q}^{*}$. See Warne (1993).
} 
Estimation of the common long memory factors and persistentnon persistent decomposition A persistent-non persistent decomposition (P-NP decomposition) of the observed variables can be performed through the decomposition of Kasa (1992), which can be written as

$$
\begin{aligned}
\mathbf{x}_{t} & =\Theta \boldsymbol{\mu}_{t}+\mathbf{u}_{t} \\
\boldsymbol{\mu}_{t} & =\left(\Theta^{\prime} \Theta\right)^{-1} \Theta^{\prime} \mathbf{x}_{t} \\
\mathbf{u}_{t} & =\boldsymbol{\beta}\left(\boldsymbol{\beta}^{\prime} \boldsymbol{\beta}\right)^{-1} \boldsymbol{\beta}^{\prime} \mathbf{x}_{t}
\end{aligned}
$$

where $\Theta\left(\Theta^{\prime} \Theta\right)^{-1} \Theta^{\prime} \mathbf{x}_{t}$ is the persistent (long memory component) and $\boldsymbol{\beta}\left(\boldsymbol{\beta}^{\prime} \boldsymbol{\beta}\right)^{-1} \boldsymbol{\beta}^{\prime} \mathbf{x}$ is the non persistent $(\mathrm{I}(0))$ component or the less persistent $\mathrm{I}(b)$ component $b>0, d-b>0$, when $\mathbf{u}_{t} \sim I(b) .{ }^{10}$ This decomposition has the important advantage of being implemented using the observed series and is suitable also for the case of fractionally cointegrated $\mathrm{I}(d)$ processes. In fact, the decomposition follows from the projection theorem, and it is always valid provided that, given a vector $\mathbf{x}_{t}$ in $R^{p}$, a closed subspace $\Theta$ of $R^{p}$ is available. Then the vector $\mathbf{x}_{t}$ can be decomposed in the sum of its projections on $\Theta$ and $\Theta_{\perp}$, where the projection operators are $\Theta\left(\Theta^{\prime} \Theta\right)^{-1} \Theta^{\prime}$ and $\boldsymbol{\beta}\left(\boldsymbol{\beta}^{\prime} \boldsymbol{\beta}\right)^{-1} \boldsymbol{\beta}^{\prime}$ (see Kasa, 1992). ${ }^{11}$

\section{The frequency domain principal components estimator: inter-}

pretation Further insights on the interpretation of the proposed estimator of the cointegration space can be gauged by noting that the principal components approach and the FDLS (frequency domain least squares) estimator can be understood in terms of orthogonal regression theory. ${ }^{12}$ Note in fact that $2 \pi \mathbf{f}(0)=\Theta \Theta^{\prime}$, and that the orthogonal regression problem can be written as

\footnotetext{
${ }^{10}$ The $\mathbf{u}_{t}$ vector is $\mathrm{I}(b)$ when the cointegrating residuals are $\mathrm{I}(b)$ or when the largest order of fractional integration of the cointegrating residuals is $\mathrm{I}(b)$. Note in fact that the $\mathbf{u}_{t}$ vector is computed as a linear combination of the cointegrating residuals.

${ }^{11}$ There are more differences than similarities between our approach and the approach of Forni et al. (2000). While both approaches rely on frequency domain principal components estimation, we focuses on the zero frequency only, and shows that the estimator retrieves the cointegration space and the factor loading matrix. Also the approach followed to identify and estimate the common factors is different, since we exploit time domain methods, which are adapted to the frequency domain. Moreover, the use of the Kasa (1992) decomposition allows the extraction of the common long memory factors from the series, which would not be achieved by applying the Forni et al. (2000) approach to fractionally differenced processes.

${ }^{12}$ See Pollock (1978; p.88-94) for results in time domain and Brillinger $(1981 ;$ ch. 8,9,10) for frequency domain least squares and principal components estimation.
} 


$$
\begin{aligned}
& \min _{\boldsymbol{\alpha}} 2 \pi \boldsymbol{\alpha}^{\prime} \mathbf{f}(0) \boldsymbol{\alpha} \\
& \text { sub } \boldsymbol{\alpha}^{\prime} \boldsymbol{\alpha}=1
\end{aligned}
$$

yielding the eigenvalue problem

$$
[2 \pi \mathbf{f}(0)-\lambda \mathbf{I}] \boldsymbol{\alpha}=\mathbf{0}
$$

A vector $\boldsymbol{\alpha}$, solution to this problem, is an eigenvector of $2 \pi \mathbf{f}(0)$. By noting that $2 \pi \boldsymbol{\alpha}^{\prime} \mathbf{f}(0) \boldsymbol{\alpha}=\lambda$, it is immediate to verify that choosing the eigenvector associated with the smallest eigenvalues is the solution to the problem.

The same eigenvalue problem arises in principal component analysis, although the objective function is expressed as

$$
\begin{gathered}
\max _{\boldsymbol{\alpha}} 2 \pi \boldsymbol{\alpha}^{\prime} \mathbf{f}(0) \boldsymbol{\alpha} \\
\text { sub } \boldsymbol{\alpha}^{\prime} \boldsymbol{\alpha}=1
\end{gathered}
$$

The solution to the problem now requires the selection of the eigenvector $\boldsymbol{\alpha}$ associated with the largest eigenvalue. We denote the linear combination $\boldsymbol{\alpha}^{\prime} \Delta^{d} \mathbf{x}^{\prime}$ as the first "long-run" principal component of $\Delta^{d} \mathbf{x} .{ }^{13}$

Hence, in principal component analysis the eigenvectors are selected starting from the one associated with the largest eigenvalue, while the solution of the general orthogonal regression problem is obtained by selecting the eigenvectors starting from the one associated with the smallest eigenvalue.

It is straightforward now to show that the FDLS problem is a particular case of the orthogonal regression problem. For the generic cointegrating regression

$$
x_{1, t}=\alpha_{2} x_{2, t}+\ldots+\alpha_{p} x_{p, t}+\epsilon_{t}
$$

we have

\footnotetext{
${ }^{13}$ Since for an I(d) vector process $\mathbf{x}_{t} \mathbf{f}_{\mathbf{x}}(\omega) \sim \omega^{-2 d} \mathbf{G} \omega \rightarrow 0^{+}$the eigenvectors of $\mathbf{f}_{\mathbf{x}}(\omega)$ $\omega \rightarrow 0^{+}$are the same as the eigenvectors of $\mathbf{G}, \mathbf{f}(0)$, or $\Theta \Theta^{\prime}$. Therefore, $\boldsymbol{\alpha}^{\prime} \mathbf{x}^{\prime}$ is the first "long-run" principal component of $\mathbf{x}$.
} 


$$
\begin{aligned}
& \min _{\boldsymbol{\alpha}} 2 \pi \boldsymbol{\alpha}^{\prime} \mathbf{f}(0) \boldsymbol{\alpha} \\
& \text { sub } \boldsymbol{\alpha}^{\prime} \mathbf{J} \boldsymbol{\alpha}=1
\end{aligned}
$$

where $\mathbf{f}(0)$ is the spectral matrix of the fractionally differenced process $\Delta^{d} x_{i}$ $i=1, \ldots, p$ at the zero frequency, with $\mathbf{J}=\left[\begin{array}{ll}1 & \mathbf{0} \\ \mathbf{0} & \mathbf{0}\end{array}\right]$, leading to normal equations that can be expressed in the form

$$
[2 \pi \mathbf{f}(0)-\lambda \mathbf{J}] \boldsymbol{\alpha}=\mathbf{0},
$$

and, after appropriate partition, as

$$
\left(2 \pi\left[\begin{array}{cc}
\mathbf{f}_{\Delta^{d} x_{1}}(0) & \mathbf{f}_{\Delta^{d} x_{1 ;} \Delta^{d} x_{2}, \ldots, \Delta^{d} x_{p}}(0) \\
\mathbf{f}_{\Delta^{d} x_{1 ;} \Delta^{d} x_{2}, \ldots, \Delta^{d} x_{p}}^{\prime}(0) & \mathbf{f}_{\Delta^{d} x_{2, \ldots, \Delta^{d} x_{p}}(0)}
\end{array}\right]-\lambda\left[\begin{array}{ll}
1 & \mathbf{0} \\
\mathbf{0} & \mathbf{0}
\end{array}\right]\right)\left[\begin{array}{c}
1 \\
-\boldsymbol{\alpha}_{*, i}
\end{array}\right]=\mathbf{0}
$$

yielding

$$
\boldsymbol{\alpha}_{*, i}=\left(\mathbf{f}_{\Delta^{d} x_{2, \ldots,}, \Delta^{d} x_{p}}(0)\right)^{-1} \mathbf{f}_{\Delta^{d} x_{1 ;} \Delta^{d} x_{2}, \ldots, \Delta^{d} x_{p}}^{\prime}(0),
$$

i.e. the FDLS estimator at the zero frequency. Note that $\lambda=2 \pi \mathbf{f}_{\Delta^{d} x_{1}}(0)-$ $2 \pi \mathbf{f}_{\Delta^{d} x_{1 ;} \Delta^{d} x_{2}, \ldots, \Delta^{d} x_{p}}(0)\left(\mathbf{f}_{\Delta^{d} x_{2, \ldots}, \Delta^{d} x_{p}}(0)\right)^{-1} \mathbf{f}_{\Delta^{d} x_{1 ;} \Delta^{d} x_{2}, \ldots, \Delta^{d} x_{p}}^{\prime}(0)$ measures the longrun variance of the fractionally differenced cointegrating residuals, which is zero since $\Delta^{d} \epsilon_{t} \sim \mathrm{I}(-\mathrm{d})$ when $\epsilon_{t} \sim \mathrm{I}(0)$, or $\mathrm{I}(\mathrm{b}-\mathrm{d})$ when $\epsilon_{t} \sim \mathrm{I}(\mathrm{b}) b>0, d-b>0$.

Since in the previous section we have established that the cointegration space is estimated by the eigenvectors associated with the smallest (zero) eigenvalues, it follows that, provided the specification is known, an equivalent estimator of the cointegration space is provided by the FDLS estimator. ${ }^{14}$

Therefore, there exists an important duality between the principal components approach proposed in the paper and the FDLS estimator, with both providing the same information concerning the long-run features of the data when the cointegration space is known. However, the proposed methodology has the clear advantage of not suffering from the well known problem given by the existence of multiple cointegrating vectors, which is relevant for multivariate models, since, in general, the specification of the cointegration space is not known.

\footnotetext{
${ }^{14}$ The equivalence of working with series in fractional differences or in levels will be also shown in the subsection below.
} 
Asymptotic results Consistent estimation of the spectral matrix at the zero frequency for the fractionally differenced series can be obtained by smoothing the periodogram matrix. Many approaches have been suggested in the literature as for instance the Daniell window, the Parzen window or the Bartlett window (see Priestly, 1981, ch.6), relying on the assumption $m \rightarrow \infty \frac{m}{T} \rightarrow 0$ as $T \rightarrow \infty$, in order to provide a consistent estimate of the spectral matrix at a given frequency. As above, we denote the estimator of the long-run variance covariance matrix obtained from the fractionally differenced processes as $2 \pi \hat{\mathbf{f}}(0)$.

Robinson (1994) has shown that the averaged periodogram can provide consistent estimates of the spectral matrix in the neighborohood of the zero frequency for long memory processes. The suggested estimator is $\hat{\mathbf{f}}_{\mathbf{x}}\left(\omega_{m}\right)=\frac{2 \pi}{T} \sum_{j=1}^{m} \operatorname{Re} \mathbf{I}\left(\omega_{j}\right) \omega_{m} \rightarrow 0^{+}$, where $\mathbf{I}(\omega)$ is the periodogram matrix for the series in levels and $\omega_{j}=2 \pi j / T$, while in Robinson and Yajima $(2002) \hat{\mathbf{f}}_{\Delta^{d} \mathbf{x}}^{*}\left(\omega_{m}\right)=\frac{1}{m} \sum_{j=1}^{m} \omega_{j}^{2 d} \operatorname{Re} \mathbf{I}\left(\omega_{j}\right)=\frac{1}{2 \pi} \Theta \Theta^{\prime} \omega_{m} \rightarrow 0^{+}$, yielding a consistent estimator of the long-run variance covariance matrix computed as $2 \pi \hat{\mathbf{f}}_{\Delta^{d} \mathbf{x}}^{*}\left(\omega_{m}\right) \omega_{m} \rightarrow 0^{+}$. In both cases estimation is carried out in the neighborhood of the origin and the assumption on the bandwidth $m$ is stated as $\frac{1}{m}+\frac{m}{T} \rightarrow 0$ as $T \rightarrow \infty$ in Robinson (1994), and $\frac{1}{m}+\frac{m^{1+2 \xi} \log \left(m^{2}\right)}{T^{2 \xi}} \rightarrow 0$ $\xi \in(0,2]$ as $T \rightarrow \infty$ in Robinson and Yajima (2002).

It is interesting to show the linkage between the FDLS estimator of the cointegrating vector obtained by working on the series in levels or in fractional differences. For the cointegrating regression $y_{t}=\beta x_{t}+e_{t}$, we have

$$
\hat{\beta}_{m}=\frac{\sum_{j=1}^{m} \operatorname{Re} I_{y, x}\left(\omega_{j}\right)}{\sum_{j=1}^{m} I_{x}\left(\omega_{j}\right)} \omega_{m} \rightarrow 0^{+}
$$

for the series in levels.

As shown by Robinson and Marinucci (1998), for vector long memory processes the following representation holds $\mathbf{f}_{\mathbf{x}}\left(\omega_{j}\right) \sim \Lambda \mathbf{G} \Lambda \omega_{j} \rightarrow 0^{+}$, with $\Lambda=\operatorname{diag}\left\{\omega_{j}^{-d_{1}}, \ldots, \omega_{j}^{-d_{p}}\right\}$. Since, as shown by Robinson and Yajima (2002), $\hat{\mathbf{f}}_{\Delta^{d^{*} \mathbf{x}}}^{*}\left(\omega_{m}\right) \omega_{m} \rightarrow 0^{+}$is a consistent estimator of the matrix $\mathbf{G}$, as also $\hat{\mathbf{f}}(0)$ is, we can consistently estimate the matrix $\mathbf{f}_{\mathbf{x}}\left(\omega_{j}\right)$ as $\omega_{m}^{-2 d^{*}} \hat{\mathbf{f}}_{\Delta^{d^{*} \mathbf{x}}}^{*}\left(\omega_{m}\right)$ or $\omega_{m}^{-2 d^{*}} \hat{\mathbf{f}}(0) \omega_{m} \rightarrow 0^{+}$, where $d^{*}=\frac{\sum_{i=1}^{p} \hat{d}_{i}}{p}$, assuming that the bandwidth employed to compute $\hat{d}_{i}$ increaseas sufficiently faster than the one employed to compute the matrix $\hat{\mathbf{G}}$, so that the fact that $d^{*}$ needs to be estimated has 
no consequences on the asymptotic distribution of $\hat{\mathbf{G}}$. The FDLS estimator can then be written as

$$
\hat{\beta}_{m}^{\Delta}=\frac{\omega_{m}^{-2 d^{*}} \sum_{j=1}^{m} \omega_{j}^{2 d^{*}} \operatorname{Re} I_{y, x}\left(\omega_{j}\right)}{\omega_{m}^{-2 d^{*}} \sum_{j=1}^{m} \omega_{j}^{2 d^{*}} I_{x}\left(\omega_{j}\right)}=\frac{\sum_{j=1}^{m} \omega_{j}^{2 d^{*}} \operatorname{Re} I_{y, x}\left(\omega_{j}\right)}{\sum_{j=1}^{m} \omega_{j}^{2 d^{*}} I_{x}\left(\omega_{j}\right)} \omega_{m} \rightarrow 0^{+}
$$

or

$$
\hat{\beta}_{0}^{\Delta}=\frac{\omega_{m}^{-2 d^{*}} \hat{f}_{\Delta^{d^{*}} y, \Delta^{d^{*} x}}(0)}{\omega_{m}^{-2 d^{*}} \hat{f}_{\Delta^{d^{*} x}}(0)}=\frac{\hat{f}_{\Delta^{d^{*}} y, \Delta^{d^{*} x}}(0)}{\hat{f}_{\Delta^{d^{*} x}}(0)}
$$

for the series in differences, where $m$ in the expression for $\hat{\beta}_{0}^{\Delta}$ corresponds to the bandwidth employed by the Daniell window.

A proof of the consistency of the $\hat{\beta}_{m}^{\Delta}$ estimator can follow the one given by Robinson and Marinucci (1998) for the $\hat{\beta}_{m}$ estimator, while for $\hat{\beta}_{0}^{\Delta}$ the reference is theorems 8.6.1 and 8.7.1 in Brillinger (1981) for the case in which the the error term and the regressor are orthogonal. Analogously to Robinson

(1994), the consistency of the FDLS estimator implemented on the fractionally differenced processes can be shown to hold also when the orthogonality condition between the regressor and error term is not assumed. As $T \rightarrow \infty$, we have

$$
\operatorname{plim}\left|\hat{\beta}_{0}^{\Delta}-\beta\right| \leq \frac{\left|f_{\Delta^{d} x \Delta^{d} e}(0)\right|}{f_{\Delta^{d} x}(0)} \leq\left(\frac{f_{\Delta^{d} e}(0)}{f_{\Delta^{d} x}(0)}\right)^{1 / 2}
$$

by the Cauchy-Schwarz inequality, where $\frac{f_{\Delta^{d} x \Delta_{e}}(0)}{f_{\Delta_{x}}(0)}$ explains the inconsistency of the FDLS estimator when the orthogonality between the regressor and error term does not hold, $\Delta^{d} e$ and $\Delta^{d} x$ refer to the fractionally differenced cointegrating residuals and regressor, respectively. Since under cointegration the residual $x \sim I(0)$ or $I(b) b>0 d-b>0$ and $\Delta^{d} e \sim I(-d)$ or $\Delta^{d} e \sim$ $I(b-d)$, hence from $\hat{f}_{\Delta^{d} x \Delta^{d} e}(\omega)=\left|1-e^{-i \omega}\right|^{2 d} \hat{f}_{x, e}(\omega)=W\left(e^{-i \omega}\right) f_{\Delta^{d} e}(\omega)$, we have $\hat{f}_{\Delta^{d} x \Delta^{d} e}(0)=0$ since $f_{\Delta^{d} e}(0)=0$ (see property $i i$ ), i.e $\hat{\beta}_{0}^{\Delta} \rightarrow_{p} \beta$ as $T \rightarrow \infty$. Therefore, this result follows from the fact that the residuals from the FDLS regression in fractional differences corresponds to the fractionally 
differenced residuals from the FDLS regression in levels and hence they are an antipersisent process, which shows a zero spectrum at the zero frequency. This result can also be understood by noting that $2 \pi f_{\Delta^{d} e}(0)=\lambda_{i}$, where $\lambda_{i}$ is the eigenvalue associated with the eigenvector $\left[\begin{array}{ll}1 & -\beta\end{array}\right]$ in the FDLS problem, which is zero under the assumption of fractional cointegration.

Asymptotic results II From Theorem 9.4.4 of Brillinger (1981) it follows that

$$
\hat{\mathbf{Q}}_{i}(0) \stackrel{a}{\sim} N\left(\mathbf{Q}_{i}(0), \Upsilon_{i}\right)
$$

where $\Upsilon_{i}=\frac{1}{2 m} \lambda_{i}(0) \sum_{l \neq i} \lambda_{l}(0)\left[\lambda_{i}(0)-\lambda_{l}(0)\right]^{-2} \mathbf{Q}_{i}(0) \mathbf{Q}_{i}(0)^{\prime}$, and $m$ is the parameter associated with the spectral matrix. Since, as argued by Robinson ans Yajima (2002), Theorem 1 of Anderson (1963) and Theorem 13.5.1 of Anderson (1984) are still true in the singular case, it follows that the above asymptotic distribution should be valid for the eigenvectors associated to the non zero eigenvalues, assuming these are ordered in descending order, with $i=1, \ldots, k$. Approximate standard errors can also be computed using resampling techniques, as, for instance, the block bootstrap or the jack-knife.

\section{Additional spectral properties for vector fractionally cointegrated processes}

In this section we discuss further spectral properties of fractionally cointegrated processes at the zero frequency, providing results which may be of practical use in empirical analysis. In particular, we show that the squared multiple coherence at the zero frequency for the fractionally differenced processes assumes a unitary value, while in the bivariate case it is the squared simple coherence to assume a unitary value. ${ }^{15}$ In addition, we also find that processes that are not fractionally cointegrated show in general positive, but lower than one, multiple and simple squared coherences at the zero frequency. In the case the dependent and independent variables are driven by different long memory factors, i.e. in the case the dependent variable is orthogonal at the zero frequency to any of the regressors, the squared multiple coherence

\footnotetext{
${ }^{15}$ Granger and Weiss (1983) and Levy (2002) have shown that these results hold for the I(1) case. Granger and Weiss (1983) have also noted that the results are still valid for the $\mathrm{I}(\mathrm{d}) \mathrm{CI}(\mathrm{d}, 0)$ case.
} 
will assume a zero value, as any of the squared simple coherences. ${ }^{16}$ We also show that all the above results holds for the series in levels as the frequency tends to zero.

Since the asymptotic distribution of the squared coherences and squared multiple coherence is known, formal tests can be undertaken to determine whether the computed coherences for the fractionally differenced processes are statistically different from one or zero, and therefore whether there exists or not fractional cointegration between the series in levels, the number of such relationships and their specification.

The main results are summarised in the theorems and corollaries below.

Theorem 1 Conditions for unitary squared multiple coherence at the zero frequency Given a vector of $p$ fractionally differenced long memory $(\mathrm{I}(\mathrm{d}))$ processes, a necessary and sufficient condition for the squared multiple coherence at the zero frequency to show a unitary value is that the processes involved are fractionally cointegrated in levels.

\section{Proof.}

Let us consider a vector of $p$ fractionally differenced $\mathrm{I}(\mathrm{d})$ processes. The associated spectral density matrix can be written as

$$
\mathbf{f}(\omega)=\left[\begin{array}{cccc}
f_{\Delta^{d} x_{1}}(\omega) & f_{\Delta^{d} x_{1} \Delta^{d} x_{2}}(\omega) & \ldots & f_{\Delta^{d} x_{1} \Delta^{d} x_{p}}(\omega) \\
f_{\Delta^{d} x_{2} \Delta^{d} x_{1}}(\omega) & f_{\Delta^{d} x_{2}}(\omega) & \vdots & \vdots \\
\vdots & \vdots & \ddots & \vdots \\
f_{\Delta^{d} x_{p} \Delta^{d} x_{1}}(\omega) & \cdots & \cdots & f_{\Delta^{d} x_{p}}(\omega)
\end{array}\right]
$$

Without lack of generality, let us now consider the power decomposition for the fractionally differenced $\mathrm{I}(\mathrm{d})$ process $\Delta^{d} x_{1}$ at the zero frequency (Priestly, 1981; p.682), in terms of the other $p-1$ variables in our model:

$$
f_{\Delta^{d} x_{1}}(0)=\mathbf{f}_{\Delta^{d} x_{1}}(0)=K_{\Delta^{d} x_{1} ; \Delta^{d} x_{2}, \ldots, \Delta^{d} x_{p}}^{2}(0) \mathbf{f}_{\Delta^{d} x_{1}}(0)+\mathbf{f}_{\nu}(0)
$$

where $K_{\Delta^{d} x_{1} ; \Delta^{d} x_{2}, \ldots, \Delta^{d} x_{p}}^{2}(0)$ is the squared multiple coherence between $\Delta^{d} x_{1}$, $\Delta^{d} x_{2}, \ldots, \Delta^{d} x_{p}$ at the zero frequency

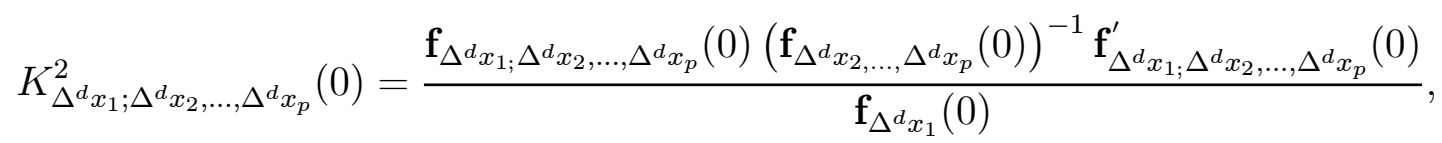

\footnotetext{
${ }^{16}$ These latter results also hold for cointegrated I(1) processes.
} 
which is the frequency domain equivalent of the time domain coefficient of determination, and the $\mathbf{f}_{i}(0)$ are appropriate submatrices of the spectral density matrix at the zero frequency $\mathbf{f}(0)$, namely:

$\mathbf{f}_{\Delta^{d} x_{1 ;} \Delta^{d} x_{2}, \ldots, \Delta^{d} x_{p}}(0)$ is the $1 \times(p-1)$ vector

$$
\left[\begin{array}{llll}
f_{\Delta^{d} x_{1} \Delta^{d} x_{2}}(0) & f_{\Delta^{d} x_{1} \Delta^{d} x_{3}}(0) \quad \ldots \quad f_{\Delta^{d} x_{1} \Delta^{d} x_{p}}(0)
\end{array}\right],
$$

$\mathbf{f}_{\Delta^{d} x_{2, \ldots}, \Delta^{d} x_{p}}(0)$ is the $(p-1) \times(p-1)$ matrix

$$
\left[\begin{array}{cccc}
f_{\Delta^{d} x_{2}}(0) & f_{\Delta^{d} x_{2} \Delta^{d} x_{3}}(0) & \ldots & f_{\Delta^{d} x_{2} \Delta^{d} x_{p}}(0) \\
f_{\Delta^{d} x_{3} \Delta^{d} x_{2}}(0) & f_{\Delta^{d} x_{3}}(0) & \vdots & \vdots \\
\vdots & \vdots & \ddots & \vdots \\
f_{\Delta^{d} x_{p} \Delta^{d} x_{2}}(0) & \ldots & \ldots & f_{\Delta^{d} x_{p}}(0)
\end{array}\right]
$$

and $\mathbf{f}_{\Delta^{d} x_{1}}(0)$ is the scalar $f_{\Delta^{d} x_{1}}(0)$.

Rearranging the expression in [25], we have

$$
1=K_{\Delta^{d} x_{1} ; \Delta^{d} x_{2}, \ldots, \Delta^{d} x_{p}}^{2}(0)+\frac{\mathbf{f}_{\nu}(0)}{\mathbf{f}_{\Delta^{d} x_{1}}(0)},
$$

which suggests that the squared multiple coherence at the zero frequency measures the proportion of power of $\Delta^{d} x_{1}$, at the zero frequency, explained by the zero frequency FDLS regression on $\Delta^{d} x_{2}, \ldots, \Delta^{d} x_{p}$, and the second term measures the residual or unexplained proportion of power. As for the coefficient of determination, the squared multiple coherence can range between zero and one, being one when the unexplained proportion of power is zero.

We can now show that fractional cointegration between the set of variables $x_{1}, x_{2}, \ldots, x_{p}$ will necessarily imply that the squared multiple coherence for $\Delta^{d} x_{1}, \Delta^{d} x_{2}, \ldots, \Delta^{d} x_{p}$ takes a unitary value.

First of all note that the residual variable $\nu$ corresponds to the fractionally differenced residuals in the fractional cointegrating regression

$$
x_{1, t}=\beta_{2} x_{2, t}+\ldots+\beta_{p} x_{p, t}+e_{t},
$$

since

$$
\Delta^{d} x_{1, t}=\beta_{2} \Delta^{d} x_{2, t}+\ldots+\beta_{p} \Delta^{d} x_{p, t}+\Delta^{d} e_{t}
$$


and hence $\nu_{t}=\Delta^{d} e_{t}$. This follows from the fact that, as shown in Section 3.1, the FDLS estimator at the zero frequency consistently estimate the known cointegration space.

Now if the set of variables $x_{1}, x_{2}, \ldots, x_{p}$ fractionally cointegrate, $e_{t} \sim I(0)$ and therefore $\nu_{t} \sim I(-d)$, or $e_{t} \sim I(b) b>0, d-b>0$ and therefore $\nu_{t} \sim I(b-d)$, hence the spectral density function at the zero frequency will assume a zero value (property $i i$ ), i.e. $\mathbf{f}_{\nu}(0)=0$, since the fractionally differenced residual is an antipersistent process.

The same result can be obtained from the interpretation provided in section 3 , by noting that $2 \pi f_{\nu}(0)=\lambda_{i}$, where $\lambda_{i}$ is one of the $p-k$ zero eigenvalues, since the zero eigenvalues measure the long-run variance of the fractionally differenced cointegrating residuals, i.e. they are the minimand in the zero fequency FDLS objective function. We can therefore also write

$$
K_{\Delta^{d} x_{1} ; \Delta^{d} x_{2}, \ldots, \Delta^{d} x_{p}}^{2}(0)=1-\frac{\lambda_{i} / 2 \pi}{\mathbf{f}_{\Delta^{d} x_{1}}(0)},
$$

Hence, from $[26,27]$ we have $K_{\Delta^{d} x_{1} ; \Delta^{d} x_{2}, \ldots, \Delta^{d} x_{p}}^{2}(0)=1$. Since in the bivariate case the squared multiple coherence and the squared coherence coincide, this result obviously holds for the bivariate case, predicting a unitary squared coherence at the zero frequency between fractionally differenced cointegrated processes.

It also follows that, if the subset of processes are not fractionally cointegrated, the squared multiple coherence will take values in the range $0 \leq$ $K_{\Delta^{d} x_{1} ; \Delta^{d} x_{2}, \ldots, \Delta^{d} x_{p}}^{2}(0)<1$, as $0 \leq K_{\Delta^{d} x_{1} ; \Delta^{d} x_{i}}^{2}(0)<1 i=2, \ldots, p$, being zero

when $\mathbf{f}_{\Delta^{d} x_{1 ;} \Delta^{d} x_{2}, \ldots, \Delta^{d} x_{p}}(0)=\mathbf{0}$, i.e. when $\Delta^{d} x_{1}$ is orthogonal to any of the regressors at the zero frequency. In fact,

$$
\begin{aligned}
K_{\Delta^{d} x_{1} ; \Delta^{d} x_{2}, \ldots, \Delta^{d} x_{p}}^{2}(0) & =\beta_{2} \frac{f_{\Delta^{d} x_{1} \Delta^{d} x_{2}}(0)}{f_{\Delta^{d} x_{1}}}+\ldots+\beta_{p} \frac{f_{\Delta^{d} x_{1} \Delta^{d} x_{p}}(0)}{f_{\Delta^{d} x_{1}}} \\
& =K_{\Delta^{d} x_{1} ; \Delta^{d} x_{2}}^{2}(0)+\ldots+K_{\Delta^{d} x_{1} ; \Delta^{d} x_{p}}^{2}(0) .
\end{aligned}
$$

This result therefore suggests that fractional cointegration between a set of $\mathrm{I}(\mathrm{d})$ processes implies and it is implied by a unitary squared multiple coherence for the fractionally differenced processes involved. An important implication of this result is that the number of unitary squared multiple coherences will be equal to the number of fractional cointegration relationships, since the theorem has established necessary and sufficient conditions. 
Note that these results hold also for the series in levels as $\omega_{j} \rightarrow 0^{+}$. In fact, we can write

$$
1=K_{x_{1} ; x_{2}, \ldots, x_{p}}^{2}\left(\omega_{j}\right)+\frac{\mathbf{f}_{e}\left(\omega_{j}\right)}{\mathbf{f}_{x_{1}}\left(\omega_{j}\right)}=K_{x_{1} ; x_{2}, \ldots, x_{p}}^{2}\left(\omega_{j}\right) \quad \omega_{j} \rightarrow 0^{+}
$$

since, in the case of cointegration $\frac{\mathbf{f}_{e}\left(\omega_{j}\right)}{\mathbf{f}_{x_{1}}\left(\omega_{j}\right)} \rightarrow 0 \quad \omega_{j} \rightarrow 0^{+}$, the denominator dominating the numerator. In fact, for the $\mathrm{CI}(d, b)$ case we have $\mathbf{f}_{e}\left(\omega_{j}\right) \sim$ $c_{e} \omega_{j}^{-2 b}$ and $\mathbf{f}_{x_{1}}\left(\omega_{j}\right) \sim c_{x_{1}} \omega_{j}^{-2 d}$ as $\omega_{j} \rightarrow 0^{+}$, hence $\frac{\mathbf{f}_{e}\left(\omega_{j}\right)}{\mathbf{f}_{x_{1}}\left(\omega_{j}\right)}=\frac{c_{e}}{c_{x_{1}}} \omega_{j}^{2(d-b)}$, while for the $\mathrm{CI}(d, 0)$ case we have $\frac{\mathbf{f}_{e}\left(\omega_{j}\right)}{\mathbf{f}_{x_{1}}\left(\omega_{j}\right)}=\frac{c_{e}}{c_{x_{1}}} \omega_{j}^{2 d}$. On the other hand, if the processes are not fractionally cointegrated we have $0 \leq K_{x_{1} ; x_{2}, \ldots, x_{p}}^{2}\left(\omega_{j}\right)<1 \quad \omega_{j} \rightarrow 0^{+}$, since $\frac{\mathbf{f}_{e}\left(\omega_{j}\right)}{\mathbf{f}_{x_{1}}\left(\omega_{j}\right)}=\frac{c_{e}}{c_{x_{1}}} \omega_{j}^{2(d-d)}=\frac{c_{e}}{c_{x_{1}}}>0$, being zero when $\mathbf{f}_{x_{1} ; x_{2}, \ldots, x_{p}}\left(\omega_{j}\right)=\mathbf{0}$, i.e. when $x_{1}$ is orthogonal to any of the regressors as $\omega_{j} \rightarrow 0^{+}$. In this latter case $\frac{\mathbf{f}_{e}\left(\omega_{j}\right)}{\mathbf{f}_{x_{1}}\left(\omega_{j}\right)}=\frac{\mathbf{f}_{x_{1}}\left(\omega_{j}\right)}{\mathbf{f}_{x_{1}}\left(\omega_{j}\right)}=1$.

The linkage between bivariate fractional cointegration and unitary squared coherences may be also established following a different approach, which leads to further insights into the spectral properties of fractionally cointegrated processes. These are discussed in the theorem and corollaries below.

Theorem 2. Conditions for unitary squared coherence at the zero frequency Given a vector of $p$ fractionally differenced long memory processes $(\mathrm{I}(d))$, a necessary and sufficient condition for the squared coherence matrix at the zero frequency to show one unitary upper (lower) diagonal element is that the two processes involved are fractionally cointegrated in levels, with bivariate cointegrating vector.

\section{Proof}

See the Appendix.

\section{Discussion}

This theorem establishes that bivariate fractional cointegration implies and it is implied by a unitary simple squared coherence for the two processes involved, following a different approach from the one used to demonstrate Theorem 1.

Corollary 2.1 The number of unitary squared coherences at the zero frequency The number of the upper (lower) diagonal unitary elements in the squared coherence matrix at the zero frequency is equal to 
$\sum_{i=1}^{k} \frac{r_{i}\left(r_{i}+1\right)}{2}$, where $r_{i}$ is the number of bivariate cointegration relationships annihilating the $i$ th factor, $\sum_{i=1}^{k} r_{i}=p-k$.

Proof

See the Appendix.

Corollary 2.2 The number of zero squared coherences at the zero frequency The number of the upper (lower) diagonal zero elements in the squared coherence matrix at the zero frequency is equal to the number of couples of long memory processes which are orthogonal at the zero frequency.

Proof

See the Appendix.

\section{Discussion}

Orthogonality at the zero frequency implies but is not implied by the lack of fractional cointegration for the processes involved. Processes which are not fractionally cointegrated will be orthogonal at the zero frequency only if the they are driven by different orthogonal common long memory factors.

Corollary 2.3 Conditions for a sparse squared coherency matrix at the zero frequency Given a vector of $p$ fractionally differenced processes, a necessary and sufficient condition for the squared coherence matrix at the zero frequency to show only unitary or zero upper (lower) diagonal elements is that each variable in levels is driven by only one common long memory factor. If the number of common long memory factors is $k<p$, and therefore the number of bivariate fractional cointegration relationships (the only to be admitted) is $r=p-k$, then the number of zero elements is in the range $\frac{(2 p-k)(k-1)}{2} \leq n_{0} \leq \frac{p(p-3)+2 k}{2}$; the number of unitary elements is in the range $p-k \leq n_{1} \leq \frac{(p-k)(p-k+1)}{2}$.

\section{Proof}

See the Appendix.

\section{Discussion}

Corollary 2.3 exploits the results of Corollary 2.1 and Corollary 2.2. It is assumed that just one long memory factor drives each process, which is common for the fractionally cointegrated processes (bivariate fractional cointegration) and it is different for the processes that are not fractionally cointegrated. 


\section{Monte Carlo results}

In the Monte Carlo exercise we have compared the small sample properties of the zero freequency FDLS estimator for the series in fractional differences and the FDLS estimator for the series in levels as the frequency tends to zero (Robinson and Marinucci, 1998). We have considered the model

$$
\begin{aligned}
y_{t} & =\beta x_{t}+e_{t} \\
\Delta^{d} x_{t} & =\varepsilon_{t} \quad d>0 \quad \varepsilon_{t} \sim N . I . D(0,1) \\
\Delta^{b} e_{t} & =v_{t}, \quad b>0, d>b \quad v_{t} \sim \text { N.I.D }(0,1) \\
E\left[\varepsilon_{t} v_{t}\right] & =\rho,
\end{aligned}
$$

and assumed three different values for the fractional differencing parameter $d(d=0.15,0.30,0.45)$, three values for the fractional differencing parameter $b(b=0,0.15,0.30)$, six values for the correlation coefficient $\rho=0,0.10$, $0.30,0.50,0.70,0.90$. The bandwidth has been set to $m=0.375 \sqrt{T}$ for both the Daniell window and the averaged periodogram, and four sample sizes have been considered $T=50,100,300,500$. The number of Monte Carlo replications for each case has been set equal to 500. ${ }^{17}$ In the simulation, in addition to the performance of the estimator of the restricted cointegration space, we have also analysed the distribution of the unitary squared coherence, and of the proportion of variance explained by the smallest eigenvalue of the spectral matrix at the zero frequency for the fractionally differenced processes and for the series in levels as the frequency tends to zero.

We have also investigated the effectivess of a bias reduction approach, based on the results

$$
\begin{gathered}
p \lim \left|\hat{\beta}_{0}^{\Delta}-\beta\right| \leq \frac{\left|f_{\Delta^{d} x \Delta^{d} e}(0)\right|}{f_{\Delta^{d} x}(0)} \leq\left(\frac{f_{\Delta^{d} e}(0)}{f_{\Delta^{d} x}(0)}\right)^{1 / 2} \\
\frac{f_{\Delta^{d} e}(0)}{f_{\Delta^{d} x}(0)}=\frac{\lambda_{0} / 2 \pi}{f_{\Delta^{d} x}(0)}
\end{gathered}
$$

where $\lambda_{0}$ is the eigenvalue of the scaled spectral matrix at the zero frequency for the fractionally differenced processess associated with the cointegrating

\footnotetext{
${ }^{17}$ The simulation of the model was performed by means of a Gauss code made available by Marc Henry, to whom the author is grateful.
} 
vector, which should be zero under the assumption of cointegration. A similar approach has been followed for the series in levels for $\omega_{j} \rightarrow 0^{+}$.

The estimated rules for bias correction are as follows

$$
\begin{aligned}
\text { bias }_{\Delta}= & \underset{(0.0332)}{-1.1263} \frac{\lambda_{0}}{f_{\Delta^{d} x}(0)}-\underset{(0.0867)}{1.1997} \frac{\lambda_{0}}{\lambda_{0}+\lambda_{1}}-\underset{(0.0204)}{0.7869}(d-b)+\underset{(0.0337)}{0.7062(d-b)^{2}} \\
& +\underset{(0.0021)}{0.0013} \ln \left(\frac{1}{T}\right)+\underset{0.0041}{0.8429} \ln \left(\frac{\lambda_{0}}{f_{\Delta^{d} x}(0)} \frac{1}{T}\right)-\underset{(0.0036)}{0.8406} \ln \left(\frac{\lambda_{0}}{\lambda_{0}+\lambda_{1}} \frac{1}{T}\right) \\
& +\underset{(0.0022)}{0.0725} \ln \left(\frac{d-b}{T}\right)+\underset{(0.0001)}{0.0040}\left(\ln \left(\frac{d-b}{T}\right)\right)^{2}
\end{aligned}
$$

for the series in differences and

$$
\begin{aligned}
& \text { bias }_{L}=\underset{(0.0423)}{0.9418} \frac{\lambda_{0}^{+}}{f_{x}\left(0^{+}\right)}-\underset{(0.1120)}{1.7371} \frac{\lambda_{0}^{+}}{\lambda_{0}^{+}+\lambda_{1}^{+}}-\underset{(0.0255)}{0.9313}(d-b)+\underset{(0.0423)}{0.8713(d-b)^{2}} \\
& +\underset{(0.0026)}{0.0051} \ln \left(\frac{1}{T}\right)+\underset{(0.0050)}{0.7937} \ln \left(\frac{\lambda_{0}^{+}}{f_{x}\left(0^{+}\right)} \frac{1}{T}\right)-\underset{(0.0044)}{0.7953 \ln }\left(\frac{\lambda_{0}^{+}}{\lambda_{0}^{+}+\lambda_{1}^{+}} \frac{1}{T}\right) \\
& +\underset{(0.0027)}{0.0628 \ln }\left(\frac{d-b}{T}\right)+\underset{(0.0001)}{0.0034}\left(\ln \left(\frac{d-b}{T}\right)\right)^{2}
\end{aligned}
$$

for the series in levels. The coefficient of determination of the two OLS regressions is close to one in both cases $\left(R_{\Delta}^{2}=0.9995\right.$ and $\left.R_{L}^{2}=0.9991\right)$, suggesting that bias correction rules may be usefully employed in practice. ${ }^{18}$

For reason of space in Tables 1-12 we have reported the results for $T=$ 100, 500, which however allow for an accurate evaluation of the performance of the FDLS estimator. ${ }^{19}$

The main findings of the Monte Carlo analysis are the following. Firstly, the FDLS estimator show the same performance independently on whether it is employed on the series in levels or in fractional differences.

Secondly, it is unbiased for moderate degrees of correlation between the cointegrating residuals and the regressor. The bias of the estimator tends to increase with the degree of correlation of the cointegrating residuals and the

\footnotetext{
${ }^{18}$ A larger number of Monte Carlo replications was employed for the estimation of the rule: 1000 replications for $T=50,100,300$ and 500 replications for $T=500$. The rule was estimated assuming $d=0.45$. The rule depends on the assumption made concerning the value of the cointegrating vector. A following work will consider a generalisation of the proposed rules for bias correction.

${ }^{19}$ The full set of results is available upon request from the author.
} 
regressor, and tends to decrease as the sample size, the spread between the fractional differencing parameter for the series and the cointegrating residuals, and the fractional differencing parameter increase. The proposed rules for bias correction are however effective, yielding an unbiased estimator in all the cases considered. On the other hand, the efficiency of the estimator tends to increase with the spread between the fractional differencing parameter of the series and the cointegrating residuals, the sample size and the degree of correlation between the cointegrating residuals and the regressor.

Thirdly, the unitary squared coherence at the zero frequency and the zero eigenvalue show a downward and upward bias, respectively. In both cases the bias tends to fall as the sample size and the spread between the fractional differencing parameter of the series and the cointegrating residual increase. On the other hand, the dependence of the bias of the unitary squared coherence and the zero eigenvalue on the degree of correlation of the regressor and the error term is related to the sign of the correlation. When the correlation is positive the bias falls as the correlation increases, while, when the correlation is negative, the bias increases as the correlation increases. This result can be understood by noting that when the correlation is positive the FDLS estimator is upward biased, so that the long-run variance of the cointegrating residual, i.e. the eigenvalue associated with the cointegrating vector, will tend to fall as the correlation increases. Conversely, when the correlation is negative the FDLS estimator is downward biased, so that the long-run variance of the cointegrating residual will tend to increase as the correlation increases. By noting that $K_{y, x}^{2}=1-\frac{\lambda / 2 \pi}{f_{y}}$, it is also possible to understand why, when the correlation is positive, the bias in the squared coherence tends to fall as the degree of correlation increases, while, when the correlation is negative, it tends to increase as the degree of correlation increases. These results suggest some caution when using fractional cointegrating rank tests as Robinson and Yajima (2002) and Phillips and Ouliaris (1988), since selecting a threshold value equal to $0.1 / p$ may lead to too conservative results. The Monte Carlo analysis therefore supports the proposed estimator, which provides a good performance also with relatively small samples.

\section{Empirical applications}

\subsection{Long memory processes}

In the empirical application with long memory processes we have studied the cointegration properties of the volatility of stock returns for four EMU 
member countries, namely France, Germany, Spain and Italy over the period January 1988- May 2000. Following Andersen et al. (1998), monthly realized variances have been computed by summing squared daily returns, yielding a total of 142 observations.

A value of the fractional differencing parameter equal to 0.36 was employed for the cointegration analysis. ${ }^{20}$ As shown in Table 13, Panel A, the Robinson and Yajima (2002) test and the Phillips and Ouliaris (1988) test provide similar results, pointing to a single large eigenvalue explaining about $80 \%$ of total variance. The remaining proportion of variance is explained by the other three eigenvalues, with the second largest one explaining about $10 \%$ of total variance. ${ }^{21}$ Since for both tests the selection of a threshold is arbitrary, we have preferred to conclude in favour of the existence of three cointegrating vectors on the basis of the estimated proportion of variance explained by each eigenvalue. ${ }^{22}$ This conclusion is also supported by the tests for unitary and zero squared coherences carried out below. Since the existence of three cointegrating vectors among four processes implies that all the identified cointegration relationships are bivariate, we expect all the off diagonal elements in the squared coherence matrix at the zero frequency to be not statistically different from one, and therefore no zero squared coherences at the zero frequency. The results of the tests for unitary and zero squared coherences are reported in Table 13, Panels B,C. As shown in the table, the null of zero squared coherence is strongly rejected for all the possible couples of variables, while the null of unitary squared coherence is never rejected for any of the possible couples at the $1 \%$ level, for the series in levels and differences. A Bonferroni bounds test for the unitary value of all the upper (lower) off diagonal elements suggests that the joint null is surely not rejected at the $5 \%$ level. The results therefore provide support for the existence of three bivariate cointegrating regressions, and validate the imposition of such identifying structure. In Table 14 the unrestricted and restricted eigenvectors are reported, the latter being the identified cointegrating and factor loading matrices. As shown in the table, there is evidence of near homogenous cointegrating vectors among the different log variance processes. From the term $\left(\Theta^{\prime} \Theta\right)^{-1} \Theta^{\prime}$ in the Kasa (1992) decomposition it is also possible to note that Germany, Italy, France and Spain have a similar weight in the determina-

\footnotetext{
${ }^{20}$ For reason of space we do not report detailed results for the persistence analysis. A full set of results is available upon request to the author.

${ }^{21}$ The bandwidth was set equal to sixteen ordinates. Qualitatively similar results have been obtained by using smaller bandwidths.

${ }^{22}$ Moreover, the Monte Carlo exercise suggests the possibility of a downward bias in the estimation of the proportion of variance explained by the non zero eigenvalues, particularly for sample sizes as the one considered in this application.
} 
tion of the common long memory factor $(0.34,0.35,0.32,0.35$ for the series in differences and $0.21,0.19,0.20,0.19$, for the series in levels) allowing to interpret the latter as a European volatility factor. Finally, in Figure 1 we plot the actual realised variance series and the estimated persistent volatility components.

\subsection{I(1) processes}

In the empirical application with I(1) processes we have studied the cointegration properties of quarterly US national accounts data, namely real GDP, real private consumption and real private investment, for the period 1947:1 through 1988:4, for a total of 168 observations. Differenced variables were expressed in percentage terms.

The results of the cointegrating rank test of Phillips and Ouliaris (1988) are reported in Table 15. For comparison we have also reported the results of the Johansen (1988) trace test.

As shown in the Table, the Phillips and Ouliaris (1988) test points to a single cointegrating vector when the threshold level is fixed at $0.10 / p$, while the Johansen (1988) test points to two cointegrating vectors linking the three variables at the $5 \%$ level. However, the proportion of variance explained by the largest eigenvalue of the spectral matrix is close to $95 \%$, allowing to conclude that just one eigenvalue of the spectral matrix is different from zero. This conclusion is supported by the squared coherence tests, since no squared coherence is statistically different from one. The estimated restricted eigenvectors are reported in Table 16. As is shown in the table, the FDLS estimator and the Johansen estimator yield similar estimates of the cointegration space, pointing to homogeneity of the cointegrating vectors. Finally, the estimated factor loading matrix obtained from the frequency domain PC approach provides similar long-run responses to the permanent shock to those obtained following the time domain approach of King et al. (1991) and Warne (1993).

\section{Conclusions}

In this paper we have proposed a new frequency domain principal components approach to the estimation of the fractional cointegration space and the factor loading matrix for the common long memory factors, which can then be recovered by the Kasa (1992) decomposition. A Monte Carlo simulation exercise suggests that the proposed estimator has good properties already 
with relatively small samples. While the estimator is proposed for fractionally cointegrated processes, the approach outlined can be also employed for I(1) cointegrated processes. Empirical applications for the two cases have been provided. Differently from the Johansen (1988) approach, the proposed methodology is potentially suited to handle also very large systems of cointegrated processes.

We have also shown that the squared multiple coherence at the zero frequency for the fractionally differenced processes assumes a unitary value, while in the bivariate case it is the squared simple coherence to assume a unitary value. These results extends the previous findings of Granger and Weiss (1983) and Levy (2002) for the I(1) case. In addition, we also find that processes that are not fractionally cointegrated show in general positive, but lower than one, multiple and simple squared coherences at the zero frequency. In the case the dependent and independent variables are driven by different long memory factors, i.e. in the case the dependent variable is orthogonal at the zero frequency to any of the regressors, the squared multiple coherence will assume a zero value, as any of the squared simple coherences. Finally, the same results hold for the series in levels as the frequency tends to zero.

\section{Appendix: proof of theorems}

The proof of the theorem and corollaries is given by making reference to a very special right null space basis of the cointegration space, which can be understood in terms of the "simple structure" of Thurstone (1947). This is the basis which contains the largest number of zero entries, and, theoretically, can always be recovered from a given basis through appropriate rotations. Therefore, despite we make reference to a specific basis, the derived results have general validity.

\section{Proof of Theorem 2: Conditions for unitary squared coherence}

at the zero frequency For the general model in [1] the squared coherence matrix $^{23}$ at the zero frequency can be written as

$$
\begin{aligned}
& \mathbf{K}^{2}(0)=\mathbf{A}(0)^{-1}\left(|\mathbf{f}(0)|^{2}\right) \mathbf{A}(0)^{-1} \\
& \mathbf{K}^{2}(0)=\mathbf{B}(0)^{-1}\left|\Theta \Theta^{\prime}\right|^{2} \mathbf{B}(0)^{-1},
\end{aligned}
$$

\footnotetext{
${ }^{23}$ We denote as squared coherence matrix the $p \times p$ matrix collecting all the possible squared coherence between the $p$ different processes. This matrix has unitary elements on the main diagonal by construction.
} 
where $\mathbf{A}(0)=\operatorname{diag}(\mathbf{f}(0))$ is a diagonal matrix containing the elements on the main diagonal in $\mathbf{f}(0)$ and $\mathbf{B}(\mathbf{0})$ is a diagonal matrix containing the elements on the main diagonal of the matrix $\Theta \Theta^{\prime}$.

Let us denote the generic element in the factor loading matrix $\Theta$ as $\theta_{i, j}$ $i=1, \ldots, p, j=1, \ldots, k$. The generic $i$ th diagonal element in $\mathbf{B}(0)$ can then be written as

$$
\sum_{l=1}^{k} \theta_{i, l}^{2} \quad i=1, \ldots, p
$$

while the generic upper diagonal element in $\left|\Theta \Theta^{\prime}\right|^{2}$ as

$$
\left|\sum_{l=1}^{k} \theta_{i, l} \theta_{j, l}\right|^{2} i=1, \ldots, p-1, \quad j=i+1, \ldots, p .
$$

The generic upper diagonal element in the squared coherence matrix $K_{i, j}^{2}(0)$ is therefore

$$
K_{i, j}^{2}(0)=\frac{\left|\sum_{l=1}^{k} \theta_{i, l} \theta_{j, l}\right|^{2}}{\sum_{l=1}^{k} \theta_{i, l}^{2} \sum_{l=1}^{k} \theta_{j, l}^{2}} i=1, \ldots, p-1, j=i+1, \ldots, p .
$$

From the Cauchy-Schwarz inequality we know that

$$
K_{i, j}^{2}(0) \leq 1
$$

In order for the Cauchy-Schwarz inequality to hold with equality it must be that

$$
\sum_{l<m}^{k} \sum^{k}\left(\theta_{i, l} \theta_{j, m}-\theta_{i, m} \theta_{j, l}\right)^{k}=0 .
$$

Now the existence of a single bivariate fractional cointegration relationship between processes $i$ and $j$ in levels implies that the $p \times k(k<p)$ simple structure for the identified factor loading matrix $\Theta$ contains two rows, $\theta_{i}$, $\theta_{j}$,with a single non zero element in the same column position, i.e. there exists only one element $s, s=1, \ldots, k$, such that $\theta_{i, s} \neq 0$ and $\theta_{j, s} \neq 0$. If this 
is the case, then all the cross products terms in [30] will be zero, and so will be the sum of their powers. We have therefore

$$
\begin{gathered}
\left|\sum_{l=1}^{k} \theta_{i, l} \theta_{j, l}\right|^{2}=\left|\theta_{i, s} \theta_{j, s}\right|^{2} \\
\sum_{l=1}^{k} \theta_{i, l}^{2} \sum_{l=1}^{k} \theta_{j, l}^{2}=\theta_{i, s}^{2} \theta_{j, s}^{2},
\end{gathered}
$$

that is

$$
K_{i, s}^{2}(0)=1
$$

Since in general $\sum_{l=1}^{k} \theta_{h, l}^{2} \sum_{l=1}^{k} \theta_{j, l}^{2}>\left|\sum_{l=1}^{k} \theta_{h, l} \theta_{j, l}\right|^{2} h \neq j, h, j \neq i, s$, all the other upper triangular elements in the squared coherence matrix will be smaller than one. Because of the symmetry of the squared coherence matrix, it will also results $K_{s, i}^{2}(0)=1$ and all the other lower diagonal entries will be smaller than one. ${ }^{24}$

\section{Proof of Corollary 2.1: The number of unitary squared coher-}

ences at the zero frequency From Theorem 2 we know that a sufficient condition for the Cauchy-Schwarz inequality to hold with equality between two generic processes $i$ and $j$ is that the corresponding rows in the factor loading matrix must contain a single non zero element in the same column position.

When more than a bivariate fractional cointegration relationship exists, the number of unitary squared coherences at the zero frequency is given by the number of combinations of class two of the row vectors satisfying the above condition. Let us denote with $r_{i} i=1, \ldots, k$ the number of cointegration relationships annihilating the $i$ th common factor. Then, the number

\footnotetext{
${ }^{24}$ Note that it could be argued that Theorem 2 establishes only a sufficient condition for a unitary squared coherence, since theoretically one could not exclude complicated non linear restrictions between the factor loadings which satisfy [30], and therefore yield a unitary coherence without requiring bivariate fractional cointegration. However, since the existence of such restrictions may be regarded as unlikely in practice, Theorem 2 establishes also necessary conditions.
} 
of combinations of row vectors satisfying Theorem 2 are $\frac{\left(r_{i}+1\right) !}{2 !\left(\left(r_{i}+1\right)-2\right) !}=$ $\frac{\left(r_{i}+1\right) !}{2 !\left(r_{i}-1\right) !}=\frac{r_{i}\left(r_{i}+1\right)}{2}$. Since there are $k$ common factors, we then have that the total number of unitary squared coherences is $\sum_{i=1}^{k} \frac{r_{i}\left(r_{i}+1\right)}{2} .{ }^{25}$

Proof of Corollary 2.2: The number of zero squared coherences at the zero frequency Note that a necessary and sufficient condition for the generic upper diagonal squared coherence at the zero frequency $K_{i, j}^{2}(0)$ to be zero is that

$$
K_{i, j}^{2}(0)=0 \leftrightarrow \sum_{l=1}^{k} \theta_{i, l} \theta_{j, l}=0 \quad i \neq j, i=1, \ldots, p-1, j=i+1, \ldots, p,
$$

i.e. a squared zero coherence at the zero frequency between two generic processes $i$ and $j$ implies, and it is implied, by the orthogonality of the subvectors containing the corresponding entries in the factor loading matrix. In the case which is most likely to hold in practice, i.e. the two processes are driven by different common long memory factors, this condition would be satisfied because the row vectors $\theta_{i}$ and $\theta_{j}$ in the simple structure for the identified factor loading matrix do not contain non zero elements in the same column position $(l)$, so that the products of their elements will always be zero, and so would be the sum of their products. Note that the orthogonality condition implies by it is not implied by the lack of fractional cointegration. Hence for $i, j=1, \ldots, p, i \neq j, l=1, \ldots, k$

$$
K_{i, j}^{2}(0)=0 \leftrightarrow \theta_{i, l}=0, \text { or } \theta_{j, l}=0 \text {, or both. }
$$

If the above condition holds, then

$$
\theta_{i, l} \theta_{j, l}=0 \quad \forall l,
$$

\footnotetext{
${ }^{25}$ Note that it could be argued that Corollary 2.1 only establishes a lower bound for the number of unitary squared coherences at the zero frequency, since theoretically one could not exclude complicated non linear restrictions between the factor loadings which satisfy [30], and therefore yield a unitary coherence without requiring bivariate fractional cointegration. However, as for Theorem 2, the existence of such restrictions may be regarded as unlikely in practice, so that Corollary 2.1 establishes the exact number of unitary squared coherences at the zero frequency.
} 
and therefore $K_{i, j}^{2}(0)=\frac{\left|\sum_{l=1}^{k} \theta_{i, l} \theta_{j, l}\right|^{2}}{\sum_{l=1}^{k} \theta_{i, l}^{2} \sum_{l=1}^{k} \theta_{j, l}^{2}}=0$.

Proof of Corollary 2.3 Conditions for a sparse squared coherency matrix at the zero frequency Given a vector of $p$ fractionally differenced processes, the number of upper diagonal elements in the squared coherence matrix at the zero frequency is $\frac{p(p-1)}{2}$. The conditions stated in the Corollary requires that the $p \times k$ simple structure for the identified factor loading matrix $\Theta$ can be partitioned, after reordering of the variables if necessary, as $\Theta=\left[\begin{array}{ll}\Theta_{1} & \Theta_{2}\end{array}\right]^{\prime}$, where $\Theta_{1}$ is diagonal with dimension equal to the number of common factors $(k<p)$, and $\Theta_{2}$ has dimension $(p-k) \times k$ and contains only row vectors with a single non zero element. Hence, the rows of the factor loading matrix satisfy either the conditions in Theorem 2 or in Corollary 2.2, and therefore all the off diagonal elements in the squared coherence matrix will be either one or zero. The number of unitary and zero coherences can be determined as follows.

To establish the number of zero squared coherences at the zero frequency let us focus on the submatrices $\Theta_{1}$ and $\Theta_{2}$ separately. Since $\Theta_{1}$ is diagonal of dimension $k$, the number of couples of vectors which satisfy the conditions in Corollary 2.2 is $\frac{k !}{2 !(k-2) !}$, i.e. the number of combinations of $k$ elements with class two, yielding $\frac{k(k-1)}{2}$ zero squared coherences coming from the elements in $\Theta_{1}$. Since by assumption there are $p-k$ bivariate cointegration relationships, and given the diagonal structure of $\Theta_{1}$, it is possible to combine each of the $(p-k)$ rows in $\Theta_{2}$ with $(k-1)$ rows in $\Theta_{1}$, so that the new $k$ rows yields a diagonal matrix. Hence, the number of zero squared coherences given by the combinations of the rows of the matrices $\Theta_{1}$ and $\Theta_{2}$ which satisfy the conditions in Corollary 2.2 is $(p-k)\left[\frac{k !}{2 !(k-2) !}-\frac{(k-1) !}{2 !(k-3) !}\right]$, where the second term in the square brackets is to avoid double accounting of the combinations of the rows in the matrix $\Theta_{1}$. By simplifying the previous expression we have $(p-k)(k-1)$.

Finally, depending on the structure of the matrix $\Theta_{2}$, the number of zero squared coherences determined by the combinations of the rows in the matrix $\Theta_{2}$ is $0 \leq q \leq \frac{(p-k) !}{2 !((p-k)-2) !}=\frac{(p-k)(p-k-1)}{2}$, with $q=0$ when all the 
rows satisfy the condition in Theorem 2 and $q=\frac{(p-k)(p-k-1)}{2}$ when all its rows satisfy Corollary 2.2 .

Therefore the number of zero squared coherences is $n_{0}=\frac{k(k-1)}{2}+$ $(p-k)(k-1)+q=\frac{(2 p-k)(k-1)}{2}+q$, that is $\frac{(2 p-k)(k-1)}{2} \leq n_{0} \leq$ $\frac{p(p-3)+2 k}{2}$.

As far as the unitary squared coherences are concerned, since the conditions in Theorem 2 hold, from Theorem 2 and Corollary 2.1 it follows that the number of unitary squared coherences will be equal to $\sum_{i=1}^{k} \frac{r_{i}\left(r_{i}+1\right)}{2}$, where $r_{i}$ is the number of bivariate cointegration relationships annihilating the $i$ th factor, and $\sum_{i=1}^{k} r_{i}=p-k$. It is now possible to note that when $q=0$, $\sum_{i=1}^{k} \frac{r_{i}\left(r_{i}+1\right)}{2}=\frac{(p-k)(p-k+1)}{2}$, and when $q=\frac{(p-k)(p-k-1)}{2}$, $\sum_{i=1}^{k} \frac{r_{i}\left(r_{i}+1\right)}{2}=p-k$. Hence $p-k \leq n_{1} \leq \frac{(p-k)(p-k+1)}{2}$.

In both cases we have $\sum_{i=1}^{k} \frac{r_{i}\left(r_{i}+1\right)}{2}+\frac{k(k-1)}{2}+(p-k)(k-1)+q=$ $\frac{p(p-1)}{2}{ }^{26}$

\section{References}

[1] Andersen, T.G., T. Bollerslev, F.X. Diebold and P. Labys, 1998, The Distribution of Exchange Rate Volatility, NBER Working paper No. 6961.

[2] Anderson, T.W., 1984, An Introduction to Multivariate Statistical Analysis, John Wiley \& Sons.

\footnotetext{
${ }^{26}$ Note that also in this case necessity follows from the exclusion of non linear restrictions between the factor loading entries which satisfy [30]. See footnotes 24 and 25 .
} 
[3] Anderson, T.W., 1963, Asymptotic Theory for Principal Components Analysis, Annals of Mathematical Statistics, 34, 122-48.

[4] Brillinger, D.R., 1981, Time Series, San Francisco: Holden Day.

[5] Engle R.F. and C.W.J., 1987, Co-Integration and Error Correction: Representation Estimation and Testing, Econometrica, 55 (2), 251-76.

[6] Forni, M., M. Hallin, M. Lippi and L. Reichlin (2000), The Generalized Dynamic-Factor Model: Identification and Estimation, The Review of Economics and Statistics, 82(4), 540-54.

[7] Granger, C.W.J and A. A. Weiss, 1983, Time Series Analysis of Error Correcting Models, in Studies in Econometrics, Time Series and Multivariate Statistics, New York: Academic Press, 255-78.

[8] Harvey, A.C., 1993, Long Memory in Stochastic Volatility, London School of Economics, Statistics Pre-print Series, LSESPS10.

[9] Johansen, S., 1988, Statistical Analysis of Cointegrating Vectors, Journal of Economic Dynamics and Control, 12, 231-54.

[10] Kasa, K., 1992, Common Stochastic Trends in International Stock Markets, Journal of Monetary Economics, 29, 95-124.

[11] King R.G., Plosser C.I., Stock J.H., and Watson M.W., 1991, Stochastic Trends and Economic Fluctuations, The American Economic Review, 81(4), 819-40.

[12] Levy, D., 2002, Cointegration in the Frequency Domain, Journal of Time Series Analysis, 23, 3, 333-339.

[13] Morana, C., 2002, Common Persistent Factors in Inflation and Excess Nominal Money Growth and a New Measure of Core Inflation, Studies in Non Linear Dynamics and Econometrics, 6(3), art.3, art.5.

[14] Phillips, P.C.B, 1986, Understanding Spurious Regressions in Econometrics, Journal of Econometrics, 33, 311-340.

[15] Phillips, P.C.B. and S. Ouliaris, 1988, Testing for Cointegration Using Principal Components Methods, Journal of Economics Dynamics and Control, 12, 205-30.

[16] Priestley, M.B., 1981, Spectral Analysis and Time Series, New York: Academic Press. 
[17] Pollock, D.S.G, 1978, The Algebra of Econometrics, John Wiley \& Sons.

[18] Robinson, P.M. and Y. Yajima, 2002, Determination of Cointegrating Rank in Fractional Systems, Journal of Econometrics, 106(2), 217-41.

[19] Robinson, P.M. and D. Marinucci, 1998, Semiparametric Frequency Domain Analysis of Fractional Cointegration, STICERD Working Paper, no. EM/98/348.

[20] Robinson, P.M., 1995, Gaussian Semiparametric Estimation of Long Range Dependence, The Annals of Statistics, 23, 1630-61.

[21] Thurstone, L.L., 1947, Multiple Factor Analysis, University of Chicago, Chicago [14.2, 14:5].

[22] Warne, A., 1993, A Common Trends Model: Identification, Estimation and Inference, Seminar Paper No. 555, IIES, Stockholm University. 
Table 1: Monte Carlo results; sample length: 100 observations, $m=4$

\begin{tabular}{ccccccc}
$d=0.15$ & \multicolumn{2}{c}{$\rho=0.0$} & \multicolumn{2}{c}{$\rho=0.1$} & \multicolumn{2}{c}{$\rho=0.3$} \\
$b=0$ & bias & mse & bias & mse & bias & mse \\
$K_{\Delta}^{2}$ & -0.388 & 0.520 & -0.324 & 0.039 & -0.288 & 0.036 \\
$K_{L}^{2}$ & -0.380 & 0.048 & -0.324 & 0.036 & -0.281 & 0.032 \\
$\beta_{\Delta}$ & -0.024 & 0.108 & 0.076 & 0.102 & 0.194 & 0.088 \\
$\beta_{L}$ & -0.024 & 0.095 & 0.076 & 0.091 & 0.195 & 0.078 \\
$\beta_{\Delta}^{c}$ & 0.022 & 0.014 & 0.008 & 0.010 & 0.010 & 0.005 \\
$\beta_{L}^{c}$ & 0.040 & 0.011 & 0.027 & 0.008 & 0.025 & 0.005 \\
$\gamma_{0}$ & 0.104 & 0.006 & 0.083 & 0.004 & 0.069 & 0.003 \\
$\gamma_{0}^{+}$ & 0.102 & 0.005 & 0.082 & 0.003 & 0.067 & 0.002 \\
& $\rho=0.5$ & $\rho$ & 0.7 & $\rho$ & 0.9 \\
$b=0$ & $b i a s$ & $m s e$ & bias & $m s e$ & bias & $m s e$ \\
$K_{\Delta}^{2}$ & -0.181 & 0.016 & -0.133 & 0.012 & -0.056 & 0.002 \\
$K_{L}^{2}$ & -0.180 & 0.015 & -0.132 & 0.012 & -0.055 & 0.002 \\
$\beta_{\Delta}$ & 0.380 & 0.062 & 0.479 & 0.050 & 0.636 & 0.021 \\
$\beta_{L}$ & 0.369 & 0.056 & 0.475 & 0.046 & 0.629 & 0.019 \\
$\beta_{\Delta}^{c}$ & -0.003 & 0.003 & -0.004 & 0.003 & -0.001 & 0.001 \\
$\beta_{L}^{c}$ & 0.014 & 0.003 & 0.016 & 0.003 & 0.018 & 0.001 \\
$\gamma_{0}$ & 0.040 & 0.001 & 0.028 & 0.001 & 0.010 & 0.000 \\
$\gamma_{0}^{+}$ & 0.040 & 0.001 & 0.028 & 0.001 & 0.010 & 0.000
\end{tabular}

The table reports the results of the Monte Carlo simulation. bias and mse denote the bias and the Monte Carlo Mean square error, respectively. $d$ is the fractional differencing operator for the series, $b$ is the fractional differencing parameter for the cointegrating residuals, $m$ is the bandwidth for the Daniell window or the averaged periodogram, $\rho$ is the correlation coefficient between the innovations of the cointegrating residuals and the regressor. $K_{i}^{2}$ is the unitary squared coherence at the zero frequency, $\beta_{i}$ is the cointegrating parameter obtained from the restricted PC estimator (FDLS estimator), where $i=\Delta$ denotes the series in fractional differences and $i=L$ denotes the series in levels. $\gamma_{0}$ and $\gamma_{0}^{+}$is the proportion of variance explained by smallest eigenvalue for the series in fractional differences and the series in levels, respectively. 
Table 2: Monte Carlo results; sample length: 100 observations, $m=4$

\begin{tabular}{ccccccc}
$d=0.30$ & \multicolumn{2}{c}{$\rho=0.0$} & \multicolumn{2}{c}{$\rho=0.1$} & \multicolumn{2}{c}{$\rho=0.3$} \\
$b=0$ & bias & mse & bias & mse & bias & mse \\
$K_{\Delta}^{2}$ & -0.251 & 0.027 & -0.246 & 0.031 & -0.184 & 0.018 \\
$K_{L}^{2}$ & -0.234 & 0.023 & -0.226 & 0.027 & -0.169 & 0.016 \\
$\beta_{\Delta}$ & 0.007 & 0.055 & 0.047 & 0.061 & 0.154 & 0.046 \\
$\beta_{L}$ & 0.009 & 0.048 & 0.051 & 0.052 & 0.146 & 0.040 \\
$\beta_{\Delta}^{c}$ & 0.022 & 0.006 & 0.026 & 0.006 & 0.014 & 0.004 \\
$\beta_{L}^{c}$ & 0.040 & 0.006 & 0.042 & 0.006 & 0.032 & 0.005 \\
$\gamma_{0}$ & 0.066 & 0.003 & 0.064 & 0.003 & 0.046 & 0.002 \\
$\gamma_{0}^{+}$ & 0.061 & 0.002 & 0.059 & 0.002 & 0.043 & 0.002 \\
& $\rho=0.5$ & $\rho$ & 0.7 & $\rho$ & 0.9 \\
$b=0$ & bias & $m s e$ & bias & $m s e$ & bias & $m s e$ \\
$K_{\Delta}^{2}$ & -0.150 & 0.014 & -0.105 & 0.006 & -0.056 & 0.002 \\
$K_{L}^{2}$ & -0.138 & 0.011 & -0.101 & 0.006 & -0.053 & 0.001 \\
$\beta_{\Delta}$ & 0.254 & 0.042 & 0.342 & 0.031 & 0.431 & 0.012 \\
$\beta_{L}$ & 0.239 & 0.036 & 0.320 & 0.025 & 0.408 & 0.010 \\
$\beta_{\Delta}^{c}$ & 0.014 & 0.003 & 0.010 & 0.002 & 0.005 & 0.001 \\
$\beta_{L}^{c}$ & 0.033 & 0.003 & 0.031 & 0.002 & 0.026 & 0.001 \\
$\gamma_{0}$ & 0.036 & 0.001 & 0.024 & 0.000 & 0.012 & 0.000 \\
$\gamma_{0}^{+}$ & 0.033 & 0.001 & 0.023 & 0.000 & 0.012 & 0.000
\end{tabular}

The table reports the results of the Monte Carlo simulation. bias and mse denote the bias and the Monte Carlo Mean square error, respectively. $d$ is the fractional differencing operator for the series, $b$ is the fractional differencing parameter for the cointegrating residuals, $m$ is the bandwidth for the Daniell window or the averaged periodogram, $\rho$ is the correlation coefficient between the innovations of the cointegrating residuals and the regressor. $K_{i}^{2}$ is the unitary squared coherence at the zero frequency, $\beta_{i}$ is the cointegrating parameter obtained from the restricted PC estimator (FDLS estimator), where $i=\Delta$ denotes the series in fractional differences and $i=L$ denotes the series in levels. $\gamma_{0}$ and $\gamma_{0}^{+}$is the proportion of variance explained by smallest eigenvalue for the series in fractional differences and the series in levels, respectively. 
Table 3: Monte Carlo results; sample length: 100 observations, $m=4$

\begin{tabular}{ccccccc}
$d=0.30$ & \multicolumn{2}{c}{$\rho=0.0$} & \multicolumn{2}{c}{$\rho=0.1$} & \multicolumn{2}{c}{$\rho=0.3$} \\
$b=0.15$ & bias & mse & bias & mse & bias & mse \\
$K_{\Delta}^{2}$ & -0.348 & 0.041 & -0.326 & 0.041 & -0.273 & 0.034 \\
$K_{L}^{2}$ & -0.338 & 0.040 & -0.314 & 0.040 & -0.263 & 0.032 \\
$\beta_{\Delta}$ & 0.008 & 0.095 & 0.064 & 0.092 & 0.205 & 0.092 \\
$\beta_{L}$ & 0.002 & 0.078 & 0.070 & 0.091 & 0.203 & 0.089 \\
$\beta_{\Delta}^{c}$ & 0.015 & 0.008 & 0.012 & 0.007 & 0.005 & 0.007 \\
$\beta_{L}^{c}$ & 0.025 & 0.009 & 0.027 & 0.009 & 0.019 & 0.008 \\
$\gamma_{0}$ & 0.092 & 0.004 & 0.085 & 0.004 & 0.066 & 0.003 \\
$\gamma_{0}^{+}$ & 0.089 & 0.004 & 0.082 & 0.004 & 0.064 & 0.003 \\
& $\rho=0.5$ & $\rho$ & 0.7 & & $\rho$ & 0.9 \\
$b=0.15$ & bias & $m s e$ & bias & mse & bias & mse \\
$K_{\Delta}^{2}$ & -0.202 & 0.021 & -0.131 & 0.009 & -0.051 & 0.002 \\
$K_{L}^{2}$ & -0.195 & 0.019 & -0.128 & 0.009 & -0.049 & 0.002 \\
$\beta_{\Delta}$ & 0.371 & 0.079 & 0.502 & 0.053 & 0.644 & 0.018 \\
$\beta_{L}$ & 0.362 & 0.068 & 0.491 & 0.047 & 0.627 & 0.018 \\
$\beta_{\Delta}^{c}$ & 0.004 & 0.004 & 0.001 & 0.003 & -0.002 & 0.001 \\
$\beta_{L}^{c}$ & 0.019 & 0.004 & 0.018 & 0.003 & 0.015 & 0.002 \\
$\gamma_{0}$ & 0.045 & 0.001 & 0.028 & 0.001 & 0.010 & 0.000 \\
$\gamma_{0}^{+}$ & 0.044 & 0.001 & 0.027 & 0.001 & 0.010 & 0.000
\end{tabular}

The table reports the results of the Monte Carlo simulation. bias and mse denote the bias and the Monte Carlo Mean square error, respectively. $d$ is the fractional differencing operator for the series, $b$ is the fractional differencing parameter for the cointegrating residuals, $m$ is the bandwidth for the Daniell window or the averaged periodogram, $\rho$ is the correlation coefficient between the innovations of the cointegrating residuals and the regressor. $K_{i}^{2}$ is the unitary squared coherence at the zero frequency, $\beta_{i}$ is the cointegrating parameter obtained from the restricted PC estimator (FDLS estimator), where $i=\Delta$ denotes the series in fractional differences and $i=L$ denotes the series in levels. $\gamma_{0}$ and $\gamma_{0}^{+}$is the proportion of variance explained by smallest eigenvalue for the series in fractional differences and the series in levels, respectively. 
Table 4: Monte Carlo results; sample length: 100 observations, $m=4$

\begin{tabular}{ccccccc}
$d=0.45$ & \multicolumn{2}{c}{$\rho=0.0$} & \multicolumn{2}{c}{$\rho=0.1$} & \multicolumn{2}{c}{$\rho=0.3$} \\
$b=0$ & bias & mse & bias & mse & bias & mse \\
$K_{\Delta}^{2}$ & -0.164 & 0.016 & -0.159 & 0.014 & -0.129 & 0.011 \\
$K_{L}^{2}$ & -0.133 & 0.011 & -0.132 & 0.010 & -0.109 & 0.008 \\
$\beta_{\Delta}$ & 0.005 & 0.033 & 0.019 & 0.030 & 0.092 & 0.023 \\
$\beta_{L}$ & 0.006 & 0.022 & 0.011 & 0.023 & 0.078 & 0.020 \\
$\beta_{\Delta}^{c}$ & 0.005 & 0.003 & 0.005 & 0.003 & 0.003 & 0.002 \\
$\beta_{L}^{c}$ & 0.014 & 0.003 & 0.014 & 0.003 & 0.015 & 0.002 \\
$\gamma_{0}$ & 0.042 & 0.001 & 0.041 & 0.001 & 0.033 & 0.008 \\
$\gamma_{0}^{+}$ & 0.034 & 0.001 & 0.034 & 0.001 & 0.028 & 0.006 \\
& $\rho=0.5$ & & $\rho$ & 0.7 & \multicolumn{2}{c}{$\rho=0.9$} \\
$b=0$ & bias & $m s e$ & bias & $m s e$ & bias & $m s e$ \\
$K_{\Delta}^{2}$ & -0.107 & 0.008 & -0.084 & 0.005 & -0.055 & 0.001 \\
$K_{L}^{2}$ & -0.092 & 0.006 & -0.074 & 0.003 & -0.049 & 0.001 \\
$\beta_{\Delta}$ & 0.156 & 0.025 & 0.206 & 0.016 & 0.276 & 0.007 \\
$\beta_{L}$ & 0.138 & 0.020 & 0.185 & 0.014 & 0.245 & 0.006 \\
$\beta_{\Delta}^{c}$ & 0.005 & 0.002 & 0.003 & 0.001 & 0.002 & 0.000 \\
$\beta_{L}^{c}$ & 0.019 & 0.002 & 0.018 & 0.001 & 0.017 & 0.001 \\
$\gamma_{0}$ & 0.028 & 0.001 & 0.021 & 0.000 & 0.013 & 0.000 \\
$\gamma_{0}^{+}$ & 0.023 & 0.001 & 0.018 & 0.000 & 0.012 & 0.000
\end{tabular}

The table reports the results of the Monte Carlo simulation. bias and mse denote the bias and the Monte Carlo Mean square error, respectively. $d$ is the fractional differencing operator for the series, $b$ is the fractional differencing parameter for the cointegrating residuals, $m$ is the bandwidth for the Daniell window or the averaged periodogram, $\rho$ is the correlation coefficient between the innovations of the cointegrating residuals and the regressor. $K_{i}^{2}$ is the unitary squared coherence at the zero frequency, $\beta_{i}$ is the cointegrating parameter obtained from the restricted PC estimator (FDLS estimator), where $i=\Delta$ denotes the series in fractional differences and $i=L$ denotes the series in levels. $\gamma_{0}$ and $\gamma_{0}^{+}$is the proportion of variance explained by smallest eigenvalue for the series in fractional differences and the series in levels, respectively. 
Table 5: Monte Carlo results; sample length: 100 observations, $m=4$

\begin{tabular}{ccccccc}
$d=0.45$ & \multicolumn{2}{c}{$\rho=0.0$} & \multicolumn{2}{c}{$\rho=0.1$} & \multicolumn{2}{c}{$\rho=0.3$} \\
$b=0.15$ & bias & mse & bias & mse & bias & mse \\
$K_{\Delta}^{2}$ & -0.243 & 0.029 & -0.226 & 0.023 & -0.188 & 0.050 \\
$K_{L}^{2}$ & -0.223 & 0.028 & -0.208 & 0.023 & -0.174 & 0.041 \\
$\beta_{\Delta}$ & 0.000 & 0.063 & 0.054 & 0.051 & 0.158 & 0.050 \\
$\beta_{L}$ & -0.003 & 0.051 & 0.040 & 0.048 & 0.135 & 0.042 \\
$\beta_{\Delta}^{c}$ & -0.014 & 0.066 & 0.016 & 0.004 & 0.015 & 0.003 \\
$\beta_{L}^{c}$ & -0.030 & 0.057 & 0.032 & 0.006 & 0.030 & 0.005 \\
$\gamma_{0}$ & 0.063 & 0.003 & 0.058 & 0.002 & 0.046 & 0.001 \\
$\gamma_{0}^{+}$ & 0.058 & 0.002 & 0.054 & 0.002 & 0.044 & 0.001 \\
& $\rho=0.5$ & $\rho$ & 0.7 & & $\rho$ & 0.9 \\
$b=0.15$ & bias & $m s e$ & bias & $m s e$ & bias & $m s e$ \\
$K_{\Delta}^{2}$ & -0.151 & 0.012 & -0.104 & 0.007 & -0.056 & 0.002 \\
$K_{L}^{2}$ & -0.136 & 0.011 & -0.094 & 0.005 & -0.051 & 0.001 \\
$\beta_{\Delta}$ & 0.250 & 0.043 & 0.334 & 0.033 & 0.431 & 0.015 \\
$\beta_{L}$ & 0.224 & 0.037 & 0.313 & 0.029 & 0.398 & 0.013 \\
$\beta_{\Delta}^{c}$ & 0.015 & 0.003 & 0.009 & 0.002 & 0.006 & 0.001 \\
$\beta_{L}^{c}$ & 0.029 & 0.003 & 0.026 & 0.002 & 0.022 & 0.001 \\
$\gamma_{0}$ & 0.036 & 0.001 & 0.024 & 0.001 & 0.012 & 0.000 \\
$\gamma_{0}^{+}$ & 0.033 & 0.001 & 0.022 & 0.000 & 0.011 & 0.000
\end{tabular}

The table reports the results of the Monte Carlo simulation. bias and mse denote the bias and the Monte Carlo Mean square error, respectively. $d$ is the fractional differencing operator for the series, $b$ is the fractional differencing parameter for the cointegrating residuals, $m$ is the bandwidth for the Daniell window or the averaged periodogram, $\rho$ is the correlation coefficient between the innovations of the cointegrating residuals and the regressor. $K_{i}^{2}$ is the unitary squared coherence at the zero frequency, $\beta_{i}$ is the cointegrating parameter obtained from the restricted PC estimator (FDLS estimator), where $i=\Delta$ denotes the series in fractional differences and $i=L$ denotes the series in levels. $\gamma_{0}$ and $\gamma_{0}^{+}$is the proportion of variance explained by smallest eigenvalue for the series in fractional differences and the series in levels, respectively. 
Table 6: Monte Carlo results; sample length: 100 observations, $m=4$

\begin{tabular}{ccccccc}
$d=0.45$ & \multicolumn{2}{c}{$\rho=0.0$} & \multicolumn{2}{c}{$\rho=0.1$} & \multicolumn{2}{c}{$\rho=0.3$} \\
$b=0.30$ & bias & mse & bias & mse & bias & mse \\
$K_{\Delta}^{2}$ & -0.352 & 0.043 & -0.329 & 0.039 & -0.258 & 0.030 \\
$K_{L}^{2}$ & -0.334 & 0.046 & -0.319 & 0.041 & -0.248 & 0.033 \\
$\beta_{\Delta}$ & 0.011 & 0.086 & 0.100 & 0.103 & 0.220 & 0.098 \\
$\beta_{L}$ & 0.019 & 0.097 & 0.090 & 0.103 & 0.216 & 0.108 \\
$\beta_{\Delta}^{c}$ & 0.017 & 0.008 & 0.010 & 0.009 & 0.004 & 0.006 \\
$\beta_{L}^{c}$ & 0.030 & 0.012 & 0.020 & 0.014 & 0.015 & 0.008 \\
$\gamma_{0}$ & 0.093 & 0.004 & 0.081 & 0.003 & 0.062 & 0.003 \\
$\gamma_{0}^{+}$ & 0.089 & 0.005 & 0.080 & 0.004 & 0.059 & 0.002 \\
& $\rho=0.5$ & $\rho$ & 0.7 & $\rho=0.9$ \\
$b=0.30$ & bias & $m s e$ & bias & $m s e$ & bias & mse \\
$K_{\Delta}^{2}$ & -0.198 & 0.020 & -0.117 & 0.007 & -0.052 & 0.003 \\
$K_{L}^{2}$ & -0.190 & 0.020 & -0.114 & 0.008 & -0.050 & 0.003 \\
$\beta_{\Delta}$ & 0.361 & 0.065 & 0.499 & 0.049 & 0.626 & 0.021 \\
$\beta_{L}$ & 0.338 & 0.070 & 0.491 & 0.053 & 0.604 & 0.023 \\
$\beta_{\Delta}^{c}$ & -0.001 & 0.003 & -0.005 & 0.003 & -0.005 & 0.001 \\
$\beta_{L}^{c}$ & 0.013 & 0.005 & 0.011 & 0.004 & 0.010 & 0.002 \\
$\gamma_{0}$ & 0.044 & 0.001 & 0.025 & 0.000 & 0.010 & 0.002 \\
$\gamma_{0}^{+}$ & 0.043 & 0.001 & 0.024 & 0.000 & 0.010 & 0.002
\end{tabular}

The table reports the results of the Monte Carlo simulation. bias and mse denote the bias and the Monte Carlo Mean square error, respectively. $d$ is the fractional differencing operator for the series, $b$ is the fractional differencing parameter for the cointegrating residuals, $m$ is the bandwidth for the Daniell window or the averaged periodogram, $\rho$ is the correlation coefficient between the innovations of the cointegrating residuals and the regressor. $K_{i}^{2}$ is the unitary squared coherence at the zero frequency, $\beta_{i}$ is the cointegrating parameter obtained from the restricted PC estimator (FDLS estimator), where $i=\Delta$ denotes the series in fractional differences and $i=L$ denotes the series in levels. $\gamma_{0}$ and $\gamma_{0}^{+}$is the proportion of variance explained by smallest eigenvalue for the series in fractional differences and the series in levels, respectively. 
Table 7: Monte Carlo results; sample length: 500 observations, $m=8$

\begin{tabular}{ccccccc}
$d=0.15$ & \multicolumn{2}{c}{$\rho=0.0$} & \multicolumn{2}{c}{$\rho=0.1$} & \multicolumn{2}{c}{$\rho=0.3$} \\
$b=0$ & bias & mse & bias & mse & bias & mse \\
$K_{\Delta}^{2}$ & -0.281 & 0.019 & -0.246 & 0.013 & -0.212 & 0.011 \\
$K_{L}^{2}$ & -0.287 & 0.016 & -0.253 & 0.012 & -0.216 & 0.010 \\
$\beta_{\Delta}$ & -0.014 & 0.045 & 0.051 & 0.025 & 0.170 & 0.029 \\
$\beta_{L}$ & -0.029 & 0.037 & 0.052 & 0.022 & 0.171 & 0.025 \\
$\beta_{\Delta}^{c}$ & 0.010 & 0.004 & 0.001 & 0.002 & 0.004 & 0.002 \\
$\beta_{L}^{c}$ & 0.026 & 0.004 & 0.017 & 0.003 & 0.019 & 0.002 \\
$\gamma_{0}$ & 0.075 & 0.002 & 0.063 & 0.001 & 0.052 & 0.001 \\
$\gamma_{0}^{+}$ & 0.076 & 0.001 & 0.065 & 0.001 & 0.053 & 0.001 \\
& $\rho=0.5$ & $\rho$ & 0.7 & $\rho$ & 0.9 \\
$b=0$ & $b i a s$ & $m s e$ & bias & $m s e$ & bias & $m s e$ \\
$K_{\Delta}^{2}$ & -0.150 & 0.006 & -0.095 & 0.003 & -0.036 & 0.000 \\
$K_{L}^{2}$ & -0.154 & 0.005 & -0.096 & 0.002 & -0.037 & 0.000 \\
$\beta_{\Delta}$ & 0.297 & 0.023 & 0.413 & 0.014 & 0.527 & 0.006 \\
$\beta_{L}$ & 0.303 & 0.020 & 0.417 & 0.012 & 0.534 & 0.005 \\
$\beta_{\Delta}^{c}$ & -0.004 & 0.001 & -0.007 & 0.001 & -0.007 & 0.000 \\
$\beta_{L}^{c}$ & 0.012 & 0.001 & 0.009 & 0.001 & 0.008 & 0.000 \\
$\gamma_{0}$ & 0.035 & 0.000 & 0.021 & 0.000 & 0.008 & 0.000 \\
$\gamma_{0}^{+}$ & 0.036 & 0.000 & 0.021 & 0.000 & 0.007 & 0.000
\end{tabular}

The table reports the results of the Monte Carlo simulation. bias and mse denote the bias and the Monte Carlo Mean square error, respectively. $d$ is the fractional differencing operator for the series, $b$ is the fractional differencing parameter for the cointegrating residuals, $m$ is the bandwidth for the Daniell window or the averaged periodogram, $\rho$ is the correlation coefficient between the innovations of the cointegrating residuals and the regressor. $K_{i}^{2}$ is the unitary squared coherence at the zero frequency, $\beta_{i}$ is the cointegrating parameter obtained from the restricted PC estimator (FDLS estimator), where $i=\Delta$ denotes the series in fractional differences and $i=L$ denotes the series in levels. $\gamma_{0}$ and $\gamma_{0}^{+}$is the proportion of variance explained by smallest eigenvalue for the series in fractional differences and the series in levels, respectively. 
Table 8: Monte Carlo results; sample length: 500 observations, $m=8$

\begin{tabular}{ccccccc}
$d=0.30$ & \multicolumn{2}{c}{$\rho=0.0$} & \multicolumn{2}{c}{$\rho=0.1$} & \multicolumn{2}{c}{$\rho=0.3$} \\
$b=0$ & bias & mse & bias & mse & bias & mse \\
$K_{\Delta}^{2}$ & -0.132 & 0.005 & -0.118 & 0.004 & -0.102 & 0.003 \\
$K_{L}^{2}$ & -0.129 & 0.004 & -0.119 & 0.004 & -0.100 & 0.002 \\
$\beta_{\Delta}$ & -0.003 & 0.012 & 0.032 & 0.012 & 0.101 & 0.010 \\
$\beta_{L}$ & 0.001 & 0.010 & 0.035 & 0.009 & 0.102 & 0.009 \\
$\beta_{\Delta}^{c}$ & -0.000 & 0.000 & -0.001 & 0.001 & 0.003 & 0.001 \\
$\beta_{L}^{c}$ & 0.009 & 0.000 & 0.014 & 0.001 & 0.017 & 0.001 \\
$\gamma_{0}$ & 0.034 & 0.000 & 0.030 & 0.000 & 0.026 & 0.000 \\
$\gamma_{0}^{+}$ & 0.033 & 0.000 & 0.030 & 0.000 & 0.025 & 0.000 \\
& $\rho=0.5$ & $\rho$ & 0.7 & $\rho$ & 0.9 \\
$b=0$ & $b i a s$ & $m s e$ & bias & $m s e$ & bias & $m s e$ \\
$K_{\Delta}^{2}$ & -0.078 & 0.002 & -0.056 & 0.001 & -0.028 & 0.000 \\
$K_{L}^{2}$ & -0.077 & 0.002 & -0.055 & 0.001 & -0.028 & 0.000 \\
$\beta_{\Delta}$ & 0.159 & 0.008 & 0.233 & 0.006 & 0.301 & 0.002 \\
$\beta_{L}$ & 0.158 & 0.007 & 0.235 & 0.005 & 0.301 & 0.002 \\
$\beta_{\Delta}^{c}$ & 0.002 & 0.000 & 0.005 & 0.000 & 0.006 & 0.000 \\
$\beta_{L}^{c}$ & 0.016 & 0.001 & 0.020 & 0.000 & 0.021 & 0.000 \\
$\gamma_{0}$ & 0.019 & 0.000 & 0.013 & 0.000 & 0.007 & 0.000 \\
$\gamma_{0}^{+}$ & 0.019 & 0.000 & 0.013 & 0.000 & 0.007 & 0.000
\end{tabular}

The table reports the results of the Monte Carlo simulation. bias and mse denote the bias and the Monte Carlo Mean square error, respectively. $d$ is the fractional differencing operator for the series, $b$ is the fractional differencing parameter for the cointegrating residuals, $m$ is the bandwidth for the Daniell window or the averaged periodogram, $\rho$ is the correlation coefficient between the innovations of the cointegrating residuals and the regressor. $K_{i}^{2}$ is the unitary squared coherence at the zero frequency, $\beta_{i}$ is the cointegrating parameter obtained from the restricted PC estimator (FDLS estimator), where $i=\Delta$ denotes the series in fractional differences and $i=L$ denotes the series in levels. $\gamma_{0}$ and $\gamma_{0}^{+}$is the proportion of variance explained by smallest eigenvalue for the series in fractional differences and the series in levels, respectively. 
Table 9: Monte Carlo results; sample length: 500 observations, $m=8$

\begin{tabular}{ccccccc}
$d=0.30$ & \multicolumn{2}{c}{$\rho=0.0$} & \multicolumn{2}{c}{$\rho=0.1$} & \multicolumn{2}{c}{$\rho=0.3$} \\
$b=0.15$ & bias & mse & bias & mse & bias & mse \\
$K_{\Delta}^{2}$ & -0.273 & 0.016 & -0.261 & 0.018 & -0.198 & 0.010 \\
$K_{L}^{2}$ & -0.275 & 0.015 & -0.260 & 0.015 & -0.197 & 0.008 \\
$\beta_{\Delta}$ & 0.001 & 0.029 & 0.051 & 0.035 & 0.169 & 0.027 \\
$\beta_{L}$ & 0.003 & 0.025 & 0.049 & 0.028 & 0.159 & 0.022 \\
$\beta_{\Delta}^{c}$ & -0.005 & 0.035 & 0.009 & 0.003 & -0.004 & 0.002 \\
$\beta_{L}^{c}$ & -0.017 & 0.033 & 0.021 & 0.003 & 0.007 & 0.002 \\
$\gamma_{0}$ & 0.072 & 0.001 & 0.068 & 0.002 & 0.049 & 0.001 \\
$\gamma_{0}^{+}$ & 0.072 & 0.001 & 0.068 & 0.001 & 0.049 & 0.001 \\
& $\rho=0.5$ & \multicolumn{2}{c}{$\rho=0.7$} & & $\rho=0.9$ \\
$b=0.15$ & bias & $m s e$ & bias & mse & bias & mse \\
$K_{\Delta}^{2}$ & -0.160 & 0.007 & -0.097 & 0.003 & -0.037 & 0.000 \\
$K_{L}^{2}$ & -0.159 & 0.006 & -0.096 & 0.003 & -0.037 & 0.000 \\
$\beta_{\Delta}$ & 0.293 & 0.024 & 0.413 & 0.016 & 0.532 & 0.006 \\
$\beta_{L}$ & 0.291 & 0.020 & 0.412 & 0.014 & 0.528 & 0.005 \\
$\beta_{\Delta}^{c}$ & 0.000 & 0.001 & -0.005 & 0.001 & -0.006 & 0.000 \\
$\beta_{L}^{c}$ & 0.013 & 0.001 & 0.008 & 0.001 & 0.007 & 0.000 \\
$\gamma_{0}$ & 0.037 & 0.000 & 0.021 & 0.001 & 0.008 & 0.000 \\
$\gamma_{0}^{+}$ & 0.037 & 0.000 & 0.021 & 0.001 & 0.008 & 0.000
\end{tabular}

The table reports the results of the Monte Carlo simulation. bias and mse denote the bias and the Monte Carlo Mean square error, respectively. $d$ is the fractional differencing operator for the series, $b$ is the fractional differencing parameter for the cointegrating residuals, $m$ is the bandwidth for the Daniell window or the averaged periodogram, $\rho$ is the correlation coefficient between the innovations of the cointegrating residuals and the regressor. $K_{i}^{2}$ is the unitary squared coherence at the zero frequency, $\beta_{i}$ is the cointegrating parameter obtained from the restricted PC estimator (FDLS estimator), where $i=\Delta$ denotes the series in fractional differences and $i=L$ denotes the series in levels. $\gamma_{0}$ and $\gamma_{0}^{+}$is the proportion of variance explained by smallest eigenvalue for the series in fractional differences and the series in levels, respectively. 
Table 10: Monte Carlo results; sample length: 500 observations, $m=8$

\begin{tabular}{ccccccc}
$d=0.45$ & \multicolumn{2}{c}{$\rho=0.0$} & \multicolumn{2}{c}{$\rho=0.1$} & \multicolumn{2}{c}{$\rho=0.3$} \\
$b=0$ & bias & mse & bias & mse & bias & mse \\
$K_{\Delta}^{2}$ & -0.058 & 0.001 & -0.053 & 0.001 & -0.048 & 0.001 \\
$K_{L}^{2}$ & -0.050 & 0.001 & -0.048 & 0.001 & -0.042 & 0.001 \\
$\beta_{\Delta}$ & -0.002 & 0.004 & 0.017 & 0.005 & 0.054 & 0.004 \\
$\beta_{L}$ & 0.001 & 0.003 & 0.018 & 0.003 & 0.054 & 0.003 \\
$\beta_{\Delta}^{c}$ & -0.001 & 0.003 & -0.000 & 0.000 & 0.004 & 0.000 \\
$\beta_{L}^{c}$ & 0.005 & 0.003 & 0.007 & 0.000 & 0.011 & 0.000 \\
$\gamma_{0}$ & 0.015 & 0.001 & 0.013 & 0.000 & 0.012 & 0.000 \\
$\gamma_{0}^{+}$ & 0.013 & 0.001 & 0.012 & 0.000 & 0.011 & 0.000 \\
& $\rho=0.5$ & $\rho$ & 0.7 & $\rho$ & 0.9 \\
$b=0$ & $b i a s$ & $m s e$ & bias & $m s e$ & bias & $m s e$ \\
$K_{\Delta}^{2}$ & -0.040 & 0.000 & -0.032 & 0.000 & -0.022 & 0.000 \\
$K_{L}^{2}$ & -0.035 & 0.000 & -0.028 & 0.000 & -0.019 & 0.000 \\
$\beta_{\Delta}$ & 0.085 & 0.003 & 0.123 & 0.003 & 0.161 & 0.001 \\
$\beta_{L}$ & 0.081 & 0.003 & 0.120 & 0.002 & 0.154 & 0.001 \\
$\beta_{\Delta}^{c}$ & 0.005 & 0.000 & 0.009 & 0.000 & 0.011 & 0.000 \\
$\beta_{L}^{c}$ & 0.013 & 0.000 & 0.017 & 0.000 & 0.019 & 0.000 \\
$\gamma_{0}$ & 0.010 & 0.000 & 0.008 & 0.000 & 0.005 & 0.000 \\
$\gamma_{0}^{+}$ & 0.010 & 0.000 & 0.007 & 0.000 & 0.005 & 0.000
\end{tabular}

The table reports the results of the Monte Carlo simulation. bias and mse denote the bias and the Monte Carlo Mean square error, respectively. $d$ is the fractional differencing operator for the series, $b$ is the fractional differencing parameter for the cointegrating residuals, $m$ is the bandwidth for the Daniell window or the averaged periodogram, $\rho$ is the correlation coefficient between the innovations of the cointegrating residuals and the regressor. $K_{i}^{2}$ is the unitary squared coherence at the zero frequency, $\beta_{i}$ is the cointegrating parameter obtained from the restricted PC estimator (FDLS estimator), where $i=\Delta$ denotes the series in fractional differences and $i=L$ denotes the series in levels. $\gamma_{0}$ and $\gamma_{0}^{+}$is the proportion of variance explained by smallest eigenvalue for the series in fractional differences and the series in levels, respectively. 
Table 11: Monte Carlo results; sample length: 100 observations, $m=4$

\begin{tabular}{ccccccc}
$d=0.45$ & \multicolumn{2}{c}{$\rho=0.0$} & \multicolumn{2}{c}{$\rho=0.1$} & \multicolumn{2}{c}{$\rho=0.3$} \\
$b=0.15$ & bias & mse & bias & mse & bias & mse \\
$K_{\Delta}^{2}$ & -0.128 & 0.005 & -0.126 & 0.005 & -0.099 & 0.003 \\
$K_{L}^{2}$ & -0.121 & 0.004 & -0.116 & 0.004 & -0.092 & 0.003 \\
$\beta_{\Delta}$ & 0.001 & 0.011 & 0.029 & 0.013 & 0.095 & 0.010 \\
$\beta_{L}$ & 0.000 & 0.010 & 0.027 & 0.010 & 0.092 & 0.008 \\
$\beta_{\Delta}^{c}$ & -0.001 & 0.001 & 0.002 & 0.001 & 0.000 & 0.001 \\
$\beta_{L}^{c}$ & 0.008 & 0.001 & 0.010 & 0.001 & 0.010 & 0.001 \\
$\gamma_{0}$ & 0.033 & 0.000 & 0.033 & 0.000 & 0.025 & 0.000 \\
$\gamma_{0}^{+}$ & 0.031 & 0.000 & 0.030 & 0.000 & 0.023 & 0.000 \\
& $\rho=0.5$ & $\rho$ & 0.7 & & $\rho$ & 0.9 \\
$b=0.15$ & bias & $m s e$ & bias & mse & bias & mse \\
$K_{\Delta}^{2}$ & -0.088 & 0.002 & -0.059 & 0.001 & -0.031 & 0.000 \\
$K_{L}^{2}$ & -0.082 & 0.002 & -0.054 & 0.001 & -0.028 & 0.000 \\
$\beta_{\Delta}$ & 0.164 & 0.010 & 0.234 & 0.007 & 0.299 & 0.003 \\
$\beta_{L}$ & 0.157 & 0.008 & 0.225 & 0.005 & 0.284 & 0.002 \\
$\beta_{\Delta}^{c}$ & 0.007 & 0.001 & 0.006 & 0.000 & 0.006 & 0.000 \\
$\beta_{L}^{c}$ & 0.018 & 0.001 & 0.018 & 0.000 & 0.017 & 0.000 \\
$\gamma_{0}$ & 0.022 & 0.000 & 0.014 & 0.000 & 0.007 & 0.000 \\
$\gamma_{0}^{+}$ & 0.020 & 0.000 & 0.013 & 0.000 & 0.007 & 0.000
\end{tabular}

The table reports the results of the Monte Carlo simulation. bias and mse denote the bias and the Monte Carlo Mean square error, respectively. $d$ is the fractional differencing operator for the series, $b$ is the fractional differencing parameter for the cointegrating residuals, $m$ is the bandwidth for the Daniell window or the averaged periodogram, $\rho$ is the correlation coefficient between the innovations of the cointegrating residuals and the regressor. $K_{i}^{2}$ is the unitary squared coherence at the zero frequency, $\beta_{i}$ is the cointegrating parameter obtained from the restricted PC estimator (FDLS estimator), where $i=\Delta$ denotes the series in fractional differences and $i=L$ denotes the series in levels. $\gamma_{0}$ and $\gamma_{0}^{+}$is the proportion of variance explained by smallest eigenvalue for the series in fractional differences and the series in levels, respectively. 
Table 12: Monte Carlo results; sample length: 500 observations, $m=8$

\begin{tabular}{ccccccc}
$d=0.45$ & \multicolumn{2}{c}{$\rho=0.0$} & \multicolumn{2}{c}{$\rho=0.1$} & \multicolumn{2}{c}{$\rho=0.3$} \\
$b=0.30$ & bias & $m s e$ & bias & mse & bias & $m s e$ \\
$K_{\Delta}^{2}$ & -0.279 & 0.018 & -0.256 & 0.015 & -0.203 & 0.011 \\
$K_{L}^{2}$ & -0.263 & 0.017 & -0.250 & 0.015 & -0.196 & 0.010 \\
$\beta_{\Delta}$ & -0.003 & 0.035 & 0.064 & 0.032 & 0.192 & 0.031 \\
$\beta_{L}$ & -0.003 & 0.032 & 0.057 & 0.030 & 0.186 & 0.027 \\
$\beta_{\Delta}^{c}$ & 0.008 & 0.003 & 0.008 & 0.003 & 0.003 & 0.002 \\
$\beta_{L}^{c}$ & 0.013 & 0.004 & 0.016 & 0.003 & 0.011 & 0.002 \\
$\gamma_{0}$ & 0.073 & 0.002 & 0.066 & 0.001 & 0.050 & 0.001 \\
$\gamma_{0}^{+}$ & 0.069 & 0.002 & 0.064 & 0.001 & 0.048 & 0.001 \\
& $\rho=0.5$ & & $\rho=0.7$ & & $\rho$ & 0.9 \\
$b=0.30$ & $b i a s$ & $m s e$ & bias & $m s e$ & bias & $m s e$ \\
$K_{\Delta}^{2}$ & -0.154 & 0.007 & -0.097 & 0.003 & -0.040 & 0.000 \\
$K_{L}^{2}$ & -0.148 & 0.007 & -0.094 & 0.003 & -0.038 & 0.000 \\
$\beta_{\Delta}$ & 0.299 & 0.027 & 0.418 & 0.021 & 0.532 & 0.007 \\
$\beta_{L}$ & 0.288 & 0.024 & 0.404 & 0.019 & 0.515 & 0.007 \\
$\beta_{\Delta}^{c}$ & -0.002 & 0.001 & -0.004 & 0.001 & -0.005 & 0.000 \\
$\beta_{L}^{c}$ & 0.007 & 0.002 & 0.005 & 0.001 & 0.004 & 0.001 \\
$\gamma_{0}$ & 0.035 & 0.000 & 0.021 & 0.000 & 0.008 & 0.000 \\
$\gamma_{0}^{+}$ & 0.035 & 0.000 & 0.021 & 0.000 & 0.008 & 0.000
\end{tabular}

The table reports the results of the Monte Carlo simulation. bias and mse denote the bias and the Monte Carlo Mean square error, respectively. $d$ is the fractional differencing operator for the series, $b$ is the fractional differencing parameter for the cointegrating residuals, $m$ is the bandwidth for the Daniell window or the averaged periodogram, $\rho$ is the correlation coefficient between the innovations of the cointegrating residuals and the regressor. $K_{i}^{2}$ is the unitary squared coherence at the zero frequency, $\beta_{i}$ is the cointegrating parameter obtained from the restricted PC estimator (FDLS estimator), where $i=\Delta$ denotes the series in fractional differences and $i=L$ denotes the series in levels. $\gamma_{0}$ and $\gamma_{0}^{+}$is the proportion of variance explained by smallest eigenvalue for the series in fractional differences and the series in levels, respectively. 
Table 13, Panel A, Fractional cointegration analysis

\begin{tabular}{llllllllll} 
RY & & \multicolumn{9}{c}{ PO } \\
eig & 0.376 & 0.045 & 0.018 & 0.011 & eig & 0.396 & 0.063 & 0.019 & 0.013 \\
$p v$ & 0.83 & 0.10 & 0.04 & 0.02 & $p v$ & 0.81 & 0.13 & 0.04 & 0.03 \\
& $1 \%$ & $5 \%$ & $10 \%$ & & & $1 \%$ & $5 \%$ & $10 \%$ & \\
$r=1$ & 0.036 & 0.032 & 0.030 & & $r=1$ & 0.038 & 0.034 & 0.032 & \\
$r=2$ & 0.100 & 0.087 & 0.082 & & $r=2$ & 0.094 & 0.085 & 0.081 & \\
$r=3$ & 0.246 & 0.223 & 0.210 & & $r=3$ & 0.283 & 0.256 & 0.242 &
\end{tabular}

Table 13, Panels B, C: zero and unitary squared coherence tests

$\begin{array}{llllllllll}\mathrm{B} & G E & F R & S P & I T & \mathrm{C} & G E & F R & S P & I T \\ G E & & 0.000 & 0.000 & 0.033 & G E & & 0.148 & 0.058 & 0.011 \\ F R & 0.000 & & 0.000 & 0.000 & F R & 0.160 & & 0.220 & 0.142 \\ S P & 0.000 & 0.000 & & 0.000 & S P & 0.136 & 0.222 & & 0.234 \\ I T & 0.000 & 0.000 & 0.000 & & I T & 0.673 & 0.172 & 0.213 & \end{array}$

Panel A reports the Robinson and Yajima (2002) (RY) and Phillips Ouliaris (1988) fractional cointegrating rank test. eig denotes the estimated eigenvalues, $p v$ the proportion of explained variance, and rank $=i$, $i=1, \ldots, 3$, denotes the corresponding test at the given significance level $(1 \%, 5 \%, 10 \%)$. The Daniell window was used for the Phillips Ouliaris test, with bandwidth set to sixteen ordinates for both tests. Panel B reports the p-values of the zero squared coherence tests. Panel $\mathrm{C}$ reports the p-values of the unitary squared coherence tests (z-trasform approach), computed according to the modified procedure suggested in Priestly (1981, p.705). Lower diagonal elements are for the series in fractional differences, upper diagonal elements are for the series in levels. 
Table 14, Unrestricted and restricted eigenvectors

\begin{tabular}{|c|c|c|c|c|}
\hline \multicolumn{5}{|c|}{ Fractional differences } \\
\hline & $\mathrm{E}_{1}$ & $\mathrm{E}_{2}$ & $\mathrm{E}_{3}$ & $\mathrm{E}_{4}$ \\
\hline$G E$ & -0.251 & 0.243 & -0.798 & 0.492 \\
\hline$F R$ & 0.909 & -0.035 & -0.042 & 0.413 \\
\hline$S P$ & -0.279 & -0.739 & 0.217 & 0.574 \\
\hline \multirow[t]{2}{*}{$I T$} & -0.181 & 0.628 & 0.561 & 0.508 \\
\hline & $G E$ & $F R$ & $S P$ & $I T$ \\
\hline \multirow{2}{*}{$G E$} & 1 & 0 & 0 & 0.742 \\
\hline & $(-)$ & $(-)$ & () & $(0.007)$ \\
\hline \multirow{2}{*}{$F R$} & -0.994 & -1.010 & 0 & 0.747 \\
\hline & $(0.009)$ & $(0.010)$ & $(-)$ & $(0.006)$ \\
\hline \multirow{2}{*}{$S P$} & 0 & 0 & 1 & 0.693 \\
\hline & $(-)$ & $(-)$ & $(-)$ & $(0.009)$ \\
\hline \multirow{2}{*}{$I T$} & 0 & 1 & -0.918 & 0.754 \\
\hline & $(-)$ & $(-)$ & $(0.010)$ & $(0.007)$ \\
\hline \multicolumn{5}{|c|}{ Levels } \\
\hline & $\mathrm{E}_{1}$ & $\mathrm{E}_{2}$ & $\mathrm{E}_{3}$ & $\mathrm{E}_{4}$ \\
\hline$G E$ & 0.294 & -0.153 & -0.803 & 0.494 \\
\hline$F R$ & -0.913 & -0.090 & -0.078 & 0.391 \\
\hline$S P$ & 0.166 & 0.714 & 0.300 & 0.610 \\
\hline \multirow[t]{2}{*}{$I T$} & 0.229 & -0.677 & 0.509 & 0.480 \\
\hline & $G E$ & $F R$ & $S P$ & $I T$ \\
\hline \multirow{2}{*}{$G E$} & 1 & 0 & 0 & 1.341 \\
\hline & $(-)$ & $(-)$ & () & $(0.012)$ \\
\hline \multirow{2}{*}{$F R$} & -1.115 & -1.014 & 0 & 1.203 \\
\hline & $(0.010)$ & (0.009) & $(-)$ & (0.001 \\
\hline \multirow{2}{*}{$S P$} & 0 & 0 & 1 & 1.243 \\
\hline & $(-)$ & $(-)$ & $(-)$ & $(0.016)$ \\
\hline \multirow{2}{*}{$I T$} & 0 & 1 & -1.019 & 1.220 \\
\hline & $(-)$ & $(-)$ & $(0.009)$ & $(0.012)$ \\
\hline
\end{tabular}

The Table reports the unrestricted (first four rows) and restricted (second four rows) eigenvectors of the scaled spectral matrix. The first three columns refer to the cointegration space, while the latter column is the factor loading matrix. Standard errors were computed using the jack-knife. 
Table 15, Panel A, Cointegration analysis

$\mathrm{J}$ $\mathrm{PO}$

$\begin{array}{llllllll}\text { eig } & 0.190 & 0.088 & 0.006 & \text { eig } & 0.693 & 0.023 & 0.008 \\ p v & 0.67 & 0.31 & 0.02 & p v & 0.96 & 0.03 & 0.01 \\ & & 95 \% & & & 1 \% & 5 \% & 10 \% \\ r=0 & 51.23 & 29.7 & & r=1 & 0.036 & 0.029 & 0.025 \\ r=1 & 16.18 & 15.4 & & r=2 & 0.139 & 0.111 & 0.096 \\ r=2 & 0.981 & 3.8 & & c v & 0.033 & 0.033 & 0.033\end{array}$

Table 15, Panels B,C: zero and unitary squared coherence tests

$\begin{array}{llllllll}\mathrm{B} & Y & C & I & \mathrm{C} & Y & C & I \\ Y & & 0.000 & 0.000 & Y & & 0.362 & 0.362 \\ C & 0.000 & & 0.000 & C & 0.295 & & 0.362 \\ I & 0.000 & 0.000 & & I & 0.354 & 0.362 & \end{array}$

Panel A reports the Johansen (1988) (J) and Phillips Ouliaris (1988) (PO) cointegrating rank test. eig denotes the estimated eigenvalues, $p v$ the proportion of explained variance, and $\operatorname{rank}=i, i=0, \ldots, 2$, denotes the corresponding test (at significance levels 1\%, 5\%, 10\% for the Phillips Ouliaris test). The Daniell window was used for the Phillips Ouliaris test, with bandwidth set to two ordinates.

Panel B reports the p-values of the zero squared coherence tests. Panel C reports the p-values of the unitary squared coherence tests (z-trasform approach), computed according to the modified procedure suggested in Priestly (1981, p.705). Lower diagonal elements are for the series in differences, upper diagonal elements are for the series in levels. 
Table 16, Unrestricted and restricted eigenvectors

\begin{tabular}{|c|c|c|c|c|c|c|c|}
\hline & $Y$ & C & $I$ & & $Y$ & $C$ & $I$ \\
\hline $\mathrm{E}_{P C, 3}^{d}$ & 0.501 & 0.250 & -0.829 & $\mathrm{~J}_{1}$ & 1 & -2.014 & 1.192 \\
\hline $\mathrm{E}_{P C, 2}^{d}$ & -0.652 & 0.739 & -0.171 & $\mathrm{~J}_{2}$ & -1.557 & 1 & 0.493 \\
\hline $\mathrm{E}_{P C, 3}^{d}$ & 0.570 & 0.626 & 0.533 & $\mathrm{~J}_{3}$ & -0.858 & -1.620 & 1 \\
\hline $\mathrm{R}_{P C, 1}^{d}$ & $\begin{array}{l}-0.966 \\
(0.015)\end{array}$ & $\begin{array}{c}1 \\
(-)\end{array}$ & $\begin{array}{c}0 \\
(-)\end{array}$ & $\mathrm{RJ}_{1}$ & $\begin{array}{l}-1.075 \\
(0.021)\end{array}$ & $\begin{array}{c}1 \\
(-)\end{array}$ & $\begin{array}{c}0 \\
(-)\end{array}$ \\
\hline $\mathrm{R}_{P C, 2}^{d}$ & $\begin{array}{l}-0.896 \\
(0.018)\end{array}$ & $\begin{array}{c}0 \\
(-)\end{array}$ & $\begin{array}{c}1 \\
(-)\end{array}$ & $\mathrm{RJ}_{2}$ & $\begin{array}{l}-0.978 \\
(0.039)\end{array}$ & $\begin{array}{c}0 \\
(-)\end{array}$ & $\begin{array}{c}1 \\
(-)\end{array}$ \\
\hline $\mathrm{R}_{P C, 3}^{d}$ & $\begin{array}{c}0.957 \\
(0.025)\end{array}$ & $\begin{array}{c}0.925 \\
(0.036)\end{array}$ & $\begin{array}{c}0.857 \\
(0.033)\end{array}$ & $\mathrm{RJ}_{3}$ & $\begin{array}{c}0.736 \\
(0.098)\end{array}$ & $\begin{array}{c}0.791 \\
(0.106)\end{array}$ & $\begin{array}{c}0.719 \\
(0.097)\end{array}$ \\
\hline $\mathrm{E}_{P C, 1}^{l}$ & 0.815 & -0.487 & -0.315 & & & & \\
\hline $\mathrm{E}_{P C, 2}^{l}$ & -0.088 & -0.641 & 0.763 & & & & \\
\hline $\mathrm{E}_{P C, 3}^{l}$ & 0.574 & 0.593 & 0.565 & & & & \\
\hline $\mathrm{R}_{P C, 1}^{l}$ & $\begin{array}{l}-1.035 \\
(0.001)\end{array}$ & $\begin{array}{c}1 \\
(-)\end{array}$ & $\begin{array}{c}0 \\
(-)\end{array}$ & & & & \\
\hline $\mathrm{R}_{P C, 2}^{l}$ & $\begin{array}{l}-0.984 \\
(0.002)\end{array}$ & $\begin{array}{c}0 \\
(-)\end{array}$ & $\begin{array}{c}1 \\
(-)\end{array}$ & & & & \\
\hline $\mathrm{R}_{P C, 3}^{l}$ & $\begin{array}{c}0.903 \\
(0.030)\end{array}$ & $\begin{array}{c}0.992 \\
(0.031)\end{array}$ & $\begin{array}{c}0.845 \\
(0.029)\end{array}$ & & & & \\
\hline
\end{tabular}

The Table reports the unrestricted (E) and restricted (R) eigenvectors of the scaled spectral matrix $(\mathrm{PC})$ and of the long-run matrix (J, Johansen (1988) estimator). $\mathrm{E}_{i}, \mathrm{R}_{i} i=1,2$ refer to the cointegration space, $\mathrm{E}_{3}, \mathrm{R}_{3}$ refer to the factor loading matrix. $\mathrm{E}_{i}^{d}, \mathrm{R}_{i}^{d} i=1,2$ refer to the cointegrating vectors obtained from the series in differences, while $\mathrm{E}_{i}^{l}, \mathrm{R}_{i}^{l} i=1,2$ refer to the cointegrating vectors obtained from the series in levels. Standard errors for the spectral matrix eigenvectors have been computed using the jack-knife. 

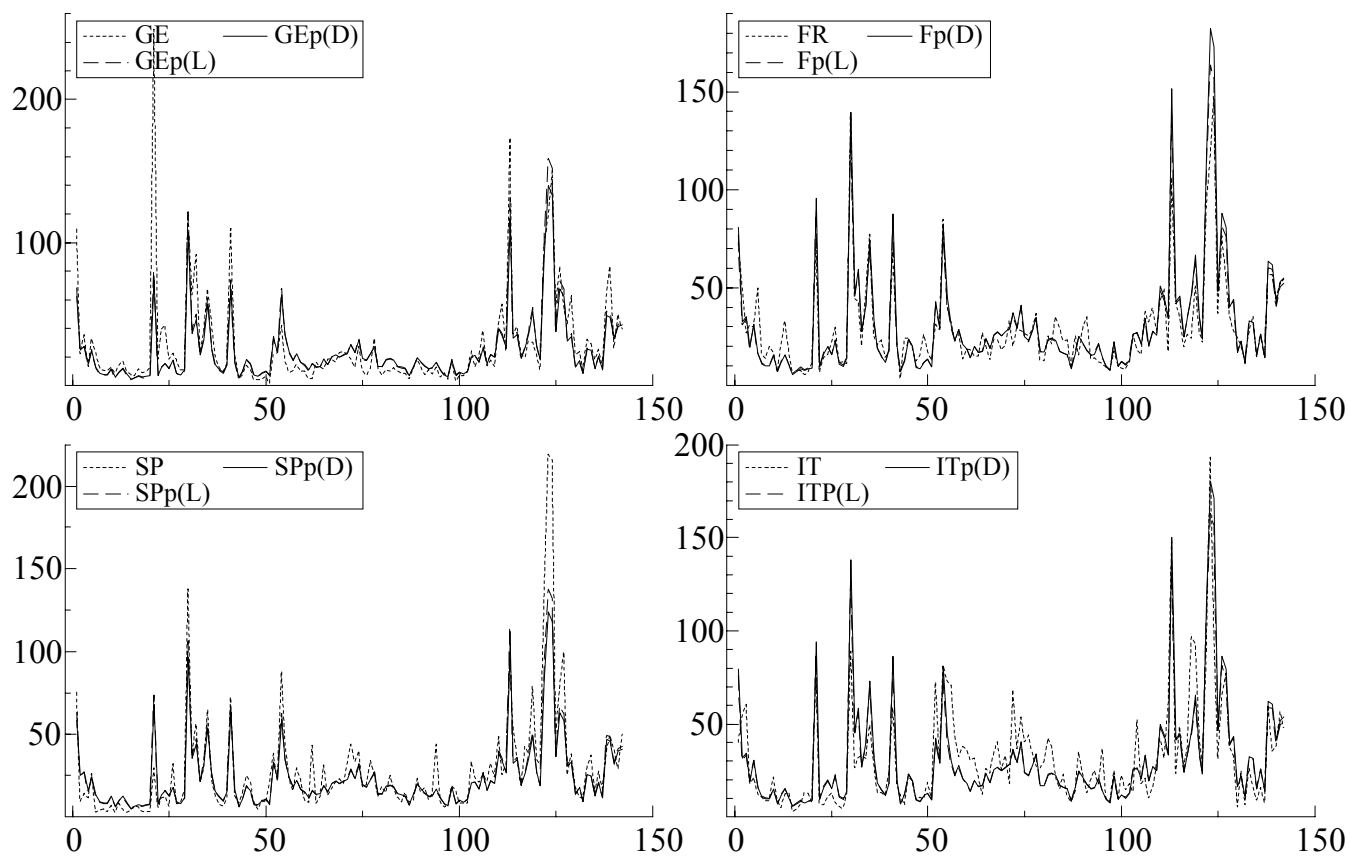

Figure 1: Actual realised variance series (Germany (GE), France (FR), Spain (SP), Italy (IT)) and estimated persistent components (P). D and L denote the decompositions obtained from the series in differences and levels. 

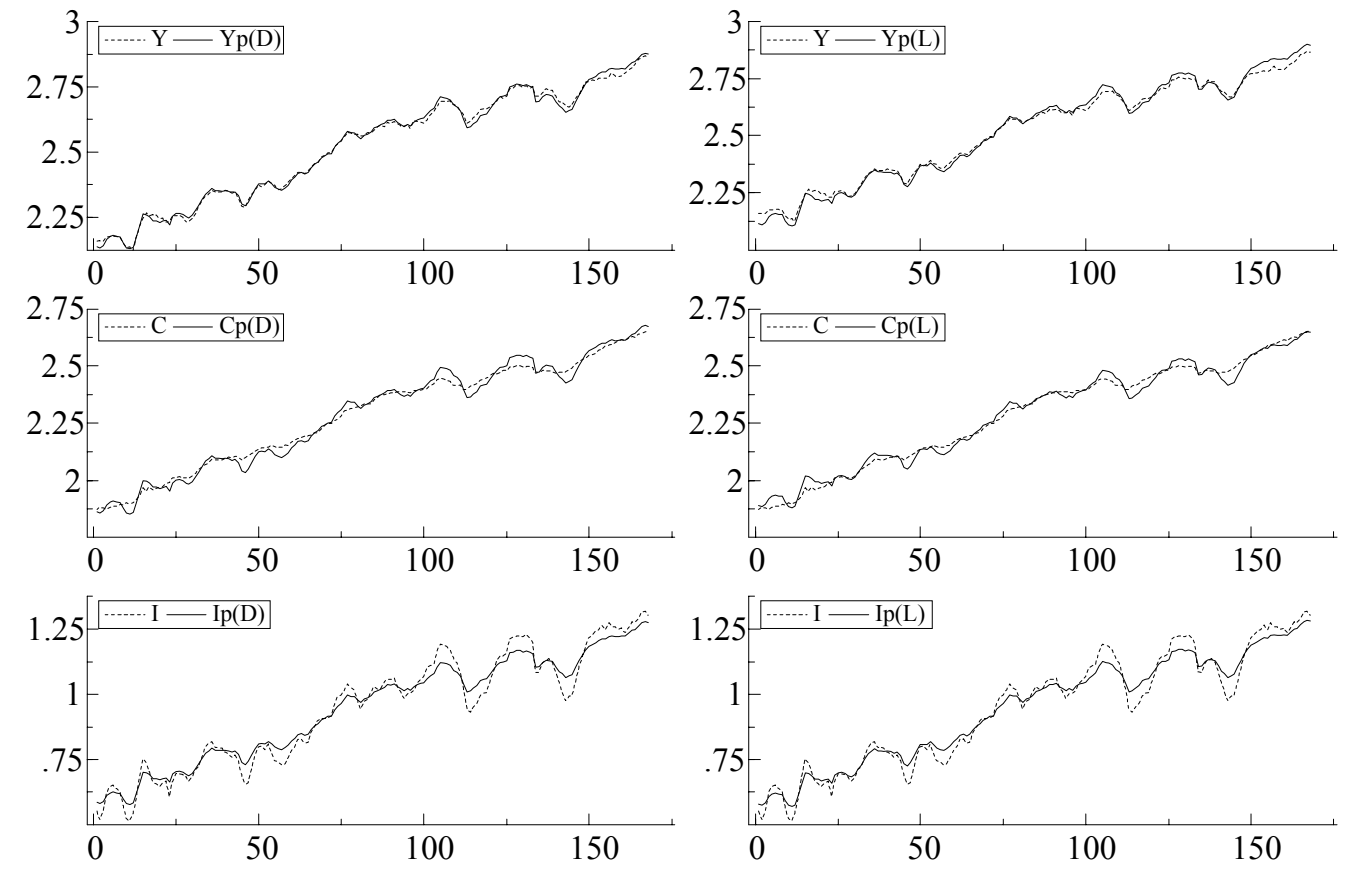

Figure 2: Actual and persistent components (GDP (Y, Yp), consumption (C, Cp), investment (I, Ip)). D and L denote the decompositions obtained from the series in differences and levels. 


\section{European Central Bank working paper series}

For a complete list of Working Papers published by the ECB, please visit the ECB's website (http://www.ecb.int).

202 "Aggregate loans to the euro area private sector" by A. Calza, M. Manrique and J. Sousa, January 2003.

203 "Myopic loss aversion, disappointment aversion and the equity premium puzzle" by D. Fielding and L. Stracca, January 2003.

204 "Asymmetric dynamics in the correlations of global equity and bond returns" by L. Cappiello, R.F. Engle and K. Sheppard, January 2003.

205 "Real exchange rate in an inter-temporal n-country-model with incomplete markets" by B. Mercereau, January 2003.

206 "Empirical estimates of reaction functions for the euro area" by D. Gerdesmeier and B. Roffia, January 2003.

207 "A comprehensive model on the euro overnight rate” by F. R. Würtz, January 2003.

208 "Do demographic changes affect risk premiums? Evidence from international data" by A. Ang and A. Maddaloni, January 2003.

209 "A framework for collateral risk control determination" by D. Cossin, Z. Huang, D. Aunon-Nerin and F. González, January 2003.

210 "Anticipated Ramsey reforms and the uniform taxation principle: the role of international financial markets” by S. Schmitt-Grohé and M. Uribe, January 2003.

2II “Self-control and savings" by P. Michel and J.P. Vidal, January 2003.

212 "Modelling the implied probability of stock market movements" by E. Glatzer and M. Scheicher, January 2003.

213 “Aggregation and euro area Phillips curves” by S. Fabiani and J. Morgan, February 2003.

2I4 “On the selection of forecasting models" by A. Inoue and L. Kilian, February 2003.

215 "Budget institutions and fiscal performance in Central and Eastern European countries" by H. Gleich, February 2003.

216 "The admission of accession countries to an enlarged monetary union: a tentative assessment” by M. Ca'Zorzi and R. A. De Santis, February 2003.

217 "The role of product market regulations in the process of structural change" by J. Messina, March 2003. 
218 "The zero-interest-rate bound and the role of the exchange rate for monetary policy in Japan" by G. Coenen and V. Wieland, March 2003.

219 "Extra-euro area manufacturing import prices and exchange rate pass-through" by B. Anderton, March 2003.

220 "The allocation of competencies in an international union: a positive analysis" by M. Ruta, April 2003.

221 "Estimating risk premia in money market rates" by A. Durré, S. Evjen and R. Pilegaard, April 2003.

222 "Inflation dynamics and subjective expectations in the United States" by K. Adam and M. Padula, April 2003.

223 "Optimal monetary policy with imperfect common knowledge" by K. Adam, April 2003.

224 "The rise of the yen vis-à-vis the ("synthetic") euro: is it supported by economic fundamentals?" by C. Osbat, R. Rüffer and B. Schnatz, April 2003.

225 "Productivity and the ("synthetic") euro-dollar exchange rate" by C. Osbat, F. Vijselaar and B. Schnatz, April 2003.

226 "The central banker as a risk manager: quantifying and forecasting inflation risks" by L. Kilian and S. Manganelli, April 2003.

227 "Monetary policy in a low pass-through environment" by T. Monacelli, April 2003.

228 "Monetary policy shocks - a nonfundamental look at the data" by M. Klaeffing, May 2003.

229 “How does the ECB target inflation?" by P. Surico, May 2003.

230 "The euro area financial system: structure, integration and policy initiatives" by P. Hartmann, A. Maddaloni and S. Manganelli, May 2003.

231 "Price stability and monetary policy effectiveness when nominal interest rates are bounded at zero" by G. Coenen, A. Orphanides and V. Wieland, May 2003.

232 "Describing the Fed's conduct with Taylor rules: is interest rate smoothing important?" by E. Castelnuovo, May 2003.

233 "The natural real rate of interest in the euro area" by N. Giammarioli and N. Valla, May 2003.

234 "Unemployment, hysteresis and transition" by M. León-Ledesma and P. McAdam, May 2003.

235 "Volatility of interest rates in the euro area: evidence from high frequency data" by N. Cassola and C. Morana, June 2003. 
236 "Swiss monetary targeting 1974-1996: the role of internal policy analysis" by G. Rich, June 2003.

237 "Growth expectations, capital flows and international risk sharing” by O. Castrén, M. Miller and R. Stiegert, June 2003.

238 "The impact of monetary union on trade prices" by R. Anderton, R. E. Baldwin and D. Taglioni, June 2003.

239 "Temporary shocks and unavoidable transitions to a high-unemployment regime" by W. J. Denhaan, June 2003.

240 "Monetary policy transmission in the euro area: any changes after EMU?" by I. Angeloni and M. Ehrmann, July 2003.

24I Maintaining price stability under free-floating: a fearless way out of the corner?" by C. Detken and V. Gaspar, July 2003.

242 "Public sector efficiency: an international comparison" by A. Afonso, L. Schuknecht and V. Tanzi, July 2003.

243 “Pass-through of external shocks to euro area inflation” by E. Hahn, July 2003.

244 "How does the ECB allot liquidity in its weekly main refinancing operations? A look at the empirical evidence" by S. Ejerskov, C. Martin Moss and L. Stracca, July 2003.

245 "Money and payments: a modern perspective" by C. Holthausen and C. Monnet, July 2003.

246 "Public finances and long-term growth in Europe - evidence from a panel data analysis" by D. R. de Ávila Torrijos and R. Strauch, July 2003.

247 "Forecasting euro area inflation: does aggregating forecasts by HICP component improve forecast accuracy?" by K. Hubrich, August 2003.

248 "Exchange rates and fundamentals" by C. Engel and K. D. West, August 2003.

249 "Trade advantages and specialisation dynamics in acceding countries" by A. Zaghini, August 2003.

250 "Persistence, the transmission mechanism and robust monetary policy" by I. Angeloni, G. Coenen and F. Smets, August 2003.

25I "Consumption, habit persistence, imperfect information and the lifetime budget constraint" by A. Willman, August 2003.

252 "Interpolation and backdating with a large information set" by E. Angelini, J. Henry and M. Marcellino, August 2003.

253 "Bond market inflation expectations and longer-term trends in broad monetary growth and inflation in industrial countries, 1880-200I” by W. G. Dewald, September 2003. 
254 "Forecasting real GDP: what role for narrow money?" by C. Brand, H.-E. Reimers and F. Seitz, September 2003.

255 "Is the demand for euro area M3 stable?" by A. Bruggeman, P. Donati and A. Warne, September 2003.

256 "Information acquisition and decision making in committees: a survey" by K. Gerling, H. P. Grüner, A. Kiel and E. Schulte, September 2003.

257 “Macroeconomic modelling of monetary policy" by M. Klaeffling, September 2003.

258 "Interest rate reaction functions and the Taylor rule in the euro area" by P. GerlachKristen, September 2003.

259 "Implicit tax co-ordination under repeated policy interactions" by M. Catenaro and J.-P. Vidal, September 2003.

260 "Aggregation-theoretic monetary aggregation over the euro area, when countries are heterogeneous" by W. A. Barnett, September 2003.

261 "Why has broad money demand been more stable in the euro area than in other economies? A literature review" by A. Calza and J. Sousa, September 2003.

262 "Indeterminacy of rational expectations equilibria in sequential financial markets" by P. Donati, September 2003.

263 "Measuring contagion with a Bayesian, time-varying coefficient model" by M. Ciccarelli and A. Rebucci, September 2003.

264 "A monthly monetary model with banking intermediation for the euro area" by A. Bruggeman and M. Donnay, September 2003.

265 "New Keynesian Phillips Curves: a reassessment using euro area data" by P. McAdam and A. Willman, September 2003.

266 "Finance and growth in the EU: new evidence from the liberalisation and harmonisation of the banking industry" by D. Romero de Ávila, September 2003.

267 "Comparing economic dynamics in the EU and CEE accession countries" by R. Süppel, September 2003.

268 "The output composition puzzle: a difference in the monetary transmission mechanism in the euro area and the US" by I. Angeloni, A. K. Kashyap, B. Mojon and D. Terlizzese, September 2003.

269 "Zero lower bound: is it a problem with the euro area?" by G. Coenen, September 2003.

270 "Downward nominal wage rigidity and the long-run Phillips curve: simulation-based evidence for the euro area" by G. Coenen, September 2003.

27I "Indeterminacy and search theory" by N. Giammarioli, September 2003. 
272 "Inflation targets and the liquidity trap" by M. Klaeffling and V. López Pérez, September 2003.

273 "Definition of price stability, range and point inflation targets: the anchoring of long-term inflation expectations” by E. Castelnuovo, S. Nicoletti-Altimari and D. RodriguezPalenzuela, September 2003.

274 "Interpreting implied risk neutral densities: the role of risk premia" by P. Hördahl and D. Vestin, September 2003.

275 "Identifying the monetary transmission mechanism using structural breaks" by A. Beyer and R. Farmer, September 2003.

276 "Short-term estimates of euro area real GDP by means of monthly data" by G. Rünstler, September 2003.

277 "On the indeterminacy of determinacy and indeterminacy" by A. Beyer and R. Farmer, September 2003.

278 "Relevant economic issues concerning the optimal rate of inflation" by D. R. Palenzuela, G. Camba-Méndez and J. Á. García, September 2003.

279 "Designing targeting rules for international monetary policy cooperation" by G. Benigno and P. Benigno, October 2003.

280 “Inflation, factor substitution and growth" by R. Klump, October 2003.

28I "Identifying fiscal shocks and policy regimes in OECD countries" by G. de Arcangelis and S. Lamartina, October 2003.

282 "Optimal dynamic risk sharing when enforcement is a decision variable" by T. V. Koeppl, October 2003.

283 "US, Japan and the euro area: comparing business-cycle features” by P. McAdam, November 2003.

284 "The credibility of the monetary policy 'free lunch"' by J. Yetman, November 2003.

285 "Government deficits, wealth effects and the price level in an optimizing model" by B. Annicchiarico, November 2003.

286 "Country and sector-specific spillover effects in the euro area, the United States and Japan" by B. Kaltenhaeuser, November 2003.

287 “Consumer inflation expectations in Poland” by T. Łyziak, November 2003.

288 "Implementing optimal control cointegrated I(I) structural VAR models" by F. V. Monti, November 2003.

289 "Monetary and fiscal interactions in open economies" by G. Lombardo and A. Sutherland, November 2003. 
290 “Inflation persistence and robust monetary policy design” by G. Coenen, November 2003.

29I “Measuring the time-inconsitency of US monetary policy” by P. Surico, November 2003.

292 "Bank mergers, competition and liquidity" by E. Carletti, P. Hartmann and G. Spagnolo, November 2003.

293 “Committees and special interests” by M. Felgenhauer and H. P. Grüner, November 2003.

294 "Does the yield spread predict recessions in the euro area?" by F. Moneta, December 2003.

295 “Optimal allotment policy in the eurosystem's main refinancing operations?" by C. Ewerhart, N. Cassola, S. Ejerskov and N. Valla, December 2003.

296 "Monetary policy analysis in a small open economy using bayesian cointegrated structural VARs?" by M. Villani and A. Warne, December 2003.

297 “Measurement of contagion in banks' equity prices” by R. Gropp and G. Moerman, December 2003.

298 "The lender of last resort: a 2 I st century approach" by X. Freixas, B. M. Parigi and J.-C. Rochet, December 2003.

299 "Import prices and pricing-to-market effects in the euro area” by T. Warmedinger, January 2004.

300 "Developing statistical indicators of the integration of the euro area banking system" by M. Manna, January 2004.

301 “Inflation and relative price asymmetry” by A. Rátfai, January 2004.

302 “Deposit insurance, moral hazard and market monitoring” by R. Gropp and J. Vesala, February 2004.

303 "Fiscal policy events and interest rate swap spreads: evidence from the EU" by A. Afonso and R. Strauch, February 2004.

304 "Equilibrium unemployment, job flows and inflation dynamics" by A. Trigari, February 2004.

305 “A structural common factor approach to core inflation estimation and forecasting" by C. Morana, February 2004.

306 "A markup model of inflation for the euro area" by C. Bowdler and E. S. Jansen, February 2004.

307 "Budgetary forecasts in Europe - the track record of stability and convergence programmes" by R. Strauch, M. Hallerberg and J. von Hagen, February 2004.

308 "International risk-sharing and the transmission of productivity shocks" by G. Corsetti, L. Dedola and S. Leduc, February 2004.

309 "Monetary policy shocks in the euro area and global liquidity spillovers" by J. Sousa and A. Zaghini, February 2004.

310 "International equity flows and returns: A quantitative equilibrium approach" by R. Albuquerque, G. H. Bauer and M. Schneider, February 2004.

311 "Current account dynamics in OECD and EU acceding countries - an intertemporal approach" by M. Bussière, M. Fratzscher and G. Müller, February 2004. 
312 "Similarities and convergence in G-7 cycles" by F. Canova, M. Ciccarelli and E. Ortega, February 2004.

313 "The high-yield segment of the corporate bond market: a diffusion modelling approach for the United States, the United Kingdom and the euro area" by G. de Bondt and D. Marqués, February 2004.

314 "Exchange rate risks and asset prices in a small open economy" by A. Derviz, March 2004.

315 "Option-implied asymmetries in bond market expectations around monetary policy actions of the ECB" by S. Vähämaa, March 2004.

316 "Cooperation in international banking supervision" by C. Holthausen and T. Rønde, March 2004.

317 "Fiscal policy and inflation volatility" by P. C. Rother, March 2004.

318 "Gross job flows and institutions in Europe" by R. Gómez-Salvador, J. Messina and G. Vallanti, March 2004.

319 "Risk sharing through financial markets with endogenous enforcement of trades" by T. V. Köppl, March 2004.

320 "Institutions and service employment: a panel study for OECD countries" by J. Messina, March 2004.

321 "Frequency domain principal components estimation of fractionally cointegrated processes" by C. Morana, March 2004. 
